\title{
Cyclin A2 maintains colon homeostasis and is a prognostic factor in colorectal cancer
}

\author{
Yuchen Guo, ${ }^{1}$ Monica Gabola, ${ }^{1}$ Rossano Lattanzio, ${ }^{2,3}$ Conception Paul, ${ }^{1}$ Valérie Pinet, ${ }^{1}$ Ruizhi Tang, ${ }^{1}$ Hulya Turali, ${ }^{1}$ \\ Julie Bremond, ${ }^{1}$ Ciro Longobardi, ${ }^{1}$ Chloé Maurizy, ${ }^{1}$ Quentin Da Costa, ${ }^{4}$ Pascal Finetti, ${ }^{4}$ Florence Boissière-Michot, ${ }^{5}$ \\ Benjamin Rivière, ${ }^{1}$ Céline Lemmers, ${ }^{6}$ Séverine Garnier, ${ }^{4}$ François Bertucci, ${ }^{4,7}$ Inti Zlobec, ${ }^{8}$ Karim Chebli, ${ }^{1}$ Jamal Tazi, \\ Rania Azar, ${ }^{9}$ Jean-Marie Blanchard, ${ }^{1}$ Peter Sicinski, ${ }^{10}$ Emilie Mamessier, ${ }^{4}$ Bénédicte Lemmers, ${ }^{1}$ and Michael Hahne ${ }^{1}$ \\ IInstitut de Génétique Moléculaire de Montpellier, Université de Montpellier, Centre National de la Recherche Scientifique (CNRS), Montpellier, France. ${ }^{2}$ Department of Medical, Oral and Biotechnological \\ Sciences, “C. d’Annunzio" University, Chieti, Italy. ${ }^{3}$ Center for Advanced Studies and Technology (CAST), “C. d’Annunzio" University, Chieti, Italy. "Predictive Oncology Laboratory, Cancer Research Center of \\ Marseille (CRCM), INSERM, U1068, CNRS, UMR7258, Institut Paoli-Calmettes, Aix Marseille Université, Marseille, France. ${ }^{5}$ Translationnal Research Unit, Montpellier Cancer Institute, Montpellier, France \\ - Université de Montpellier, Montpellier, France. ${ }^{6}$ PVM, Biocampus, Université de Montpellier, CNRS, Montpellier, France. 'Department of Medical Oncology, Institut Paoli-Calmettes, Marseille, France. \\ ${ }^{8}$ Institute of Pathology, University of Bern, Bern, Switzerland. ${ }^{9}$ Faculty of Pharmacy, Lebanese University, Hadath, Lebanon. ${ }^{10}$ Dana Farber Institute, Boston, Massachusetts, USA.
}

\begin{abstract}
To clarify the function of cyclin A2 in colon homeostasis and colorectal cancer (CRC), we generated mice deficient for cyclin A2 in colonic epithelial cells (CECs). Colons of these mice displayed architectural changes in the mucosa and signs of inflammation, as well as increased proliferation of CECs associated with the appearance of low- and high-grade dysplasias. The main initial events triggering those alterations in cyclin A2-deficient CECs appeared to be abnormal mitoses and DNA damage. Cyclin A2 deletion in CECs promoted the development of dysplasia and adenocarcinomas in a murine colitisassociated cancer model. We next explored the status of cyclin A2 expression in clinical CRC samples at the mRNA and protein levels and found higher expression in tumors of patients with stage 1 or 2 CRC compared with those of patients with stage 3 or 4 CRC. A meta-analysis of 11 transcriptome data sets comprising 2239 primary CRC tumors revealed different expression levels of CCNA2 (the mRNA coding for cyclin A2) among the CRC tumor subtypes, with the highest expression detected in consensus molecular subtype 1 (CMS1) and the lowest in CMS4 tumors. Moreover, we found high expression of CCNA2 to be a new, independent prognosis factor for CRC tumors.
\end{abstract}

\section{Introduction}

Colorectal cancer (CRC) is one of the leading causes of malignancy-related death worldwide, with an increasing incidence (1). The disease status of patients can be classified according to histologic tumor grade (differentiation) and anatomic extent of the disease (i.e., tumor/node/metastasis [TNM], stages 1-4), which describes tumor growth into the wall of the rectum or colon (stage 2), involvement of regional lymph nodes (stage 3 ), and metastatic spread to other organs (stage 4) (2). Once metastasis has developed, 5-year survival rates following surgery are below $10 \%$ (3). Also, adjuvant chemotherapy after surgical resection for stage $3 \mathrm{CRC}$ and high-risk stage 2 tumors (i.e., tumors with poor differentiation, T4 classification, lymphovascular invasion, or perineural invasion) increased patients' survival rates, but many will relapse or develop distant metastases $(4,5)$. Thus, there is an urgent need to better understand the molecular changes leading to the development of CRC to identify novel therapeutic strategies.

An important risk factor for CRC development is inflammation. Patients with early-onset chronic inflammatory bowel disease (IBD)

Authorship note: YG and MG are co-first authors. EM, BL, and MH are co-last authors. Conflict of interest: The authors have declared that no conflict of interest exists. Copyright: @ 2021, American Society for Clinical Investigation.

Submitted: July 10, 2019; Accepted: December 9, 2020; Published: February 15, 2021

Reference information: J Clin Invest. 2021;131(4):e131517.

https://doi.org/10.1172/JCI131517. and widespread manifestation (i.e., pancolitis) have a higher probability of developing CRC (6). Furthermore, inherited mutations have been identified for $5 \%$ of patients with CRC, with diseases traditionally divided into polyposis syndromes, such as the familial adenomatous polyposis (FAP) and nonpolyposis syndromes like Lynch syndrome, also known as hereditary nonpolyposis colorectal cancer (HNPCC) (7). Sporadic CRC is a molecularly highly heterogeneous disease. Research teams from different institutions recently performed a meta-analysis of the different reported subclassifications to define the existence of 4 distinct gene expression-based subtypes (consensus molecular subtypes [CMSs]), i.e., immune (CMS1), canonical (CMS2), metabolic (CMS3), and mesenchymal (CMS4) (8). Each CMS has a unique biology and gene expression pattern (8), thus providing a framework for designing subtypespecific and personalized treatments. Indeed, CMSs have different clinical features including prognosis and response to therapies, with CMS4 having the worst prognosis.

Cyclin A2 is an established regulator of cell proliferation and has been used for molecular diagnostics as a proliferation marker (9). However, several studies suggest that cell-cycle regulators can exert additional functions, well beyond cell-cycle control (10-16). For example, the cyclin CDK inhibitors p21, p27, and p57, as well as cyclin D1, have been found to be involved in the control of apoptosis, transcription, cytoskeletal dynamics as well as cell migration $(12-13,16)$ 
A
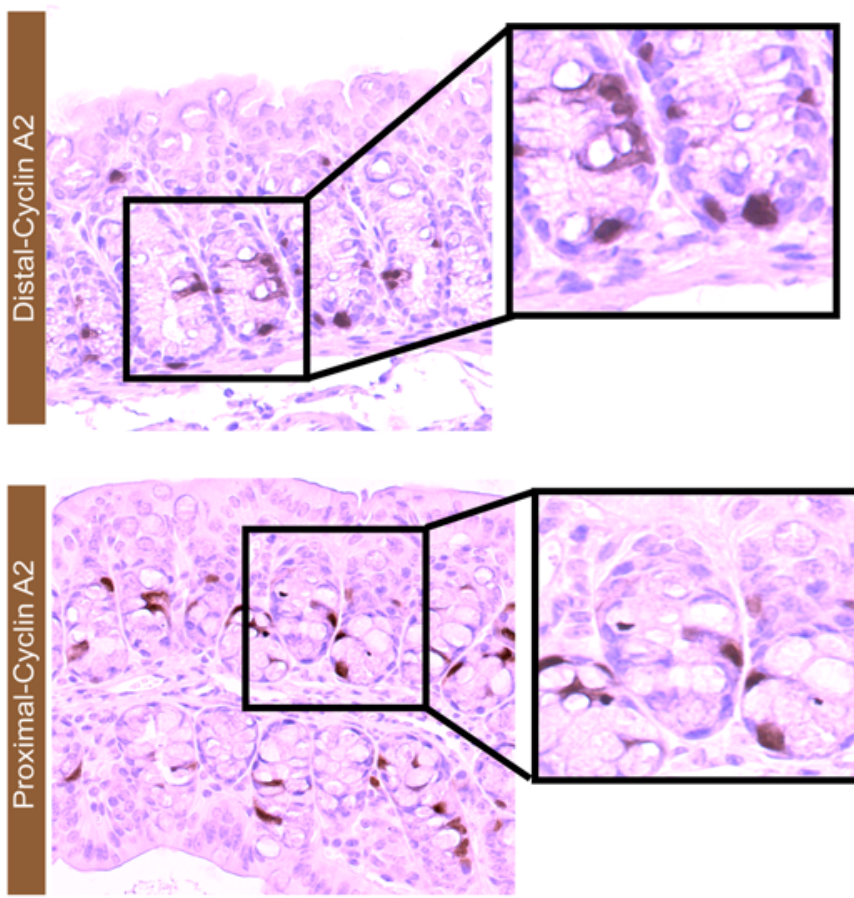

VilCreCcna2 $2^{\text {wn }}$

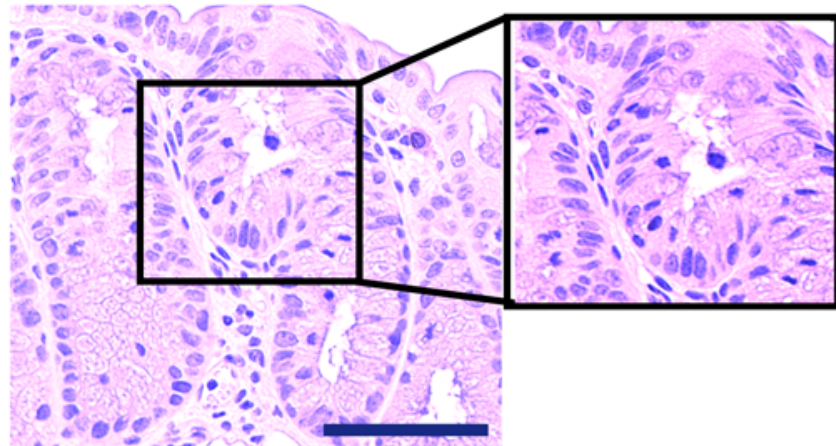

B

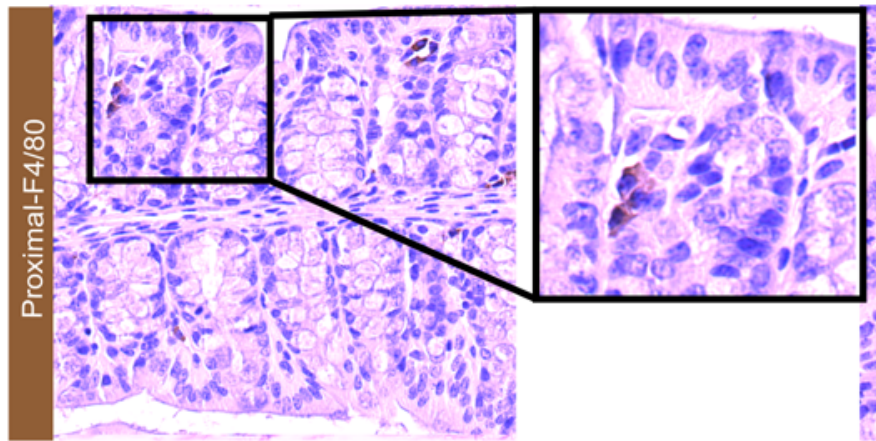

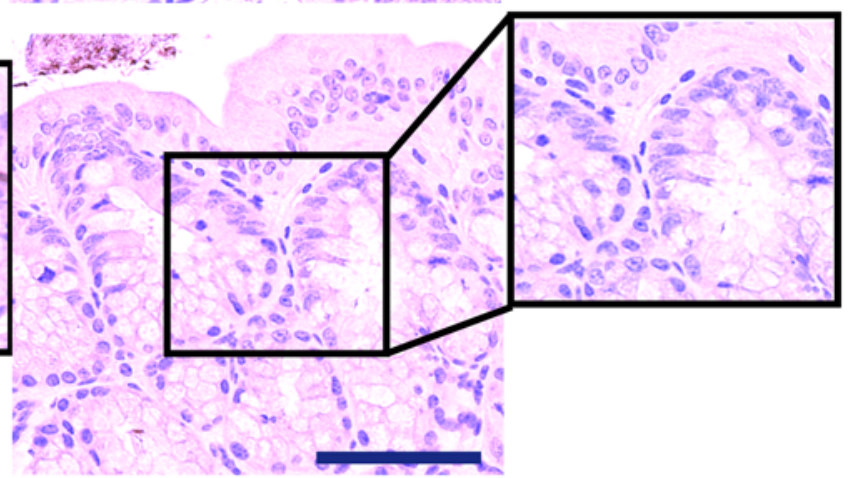

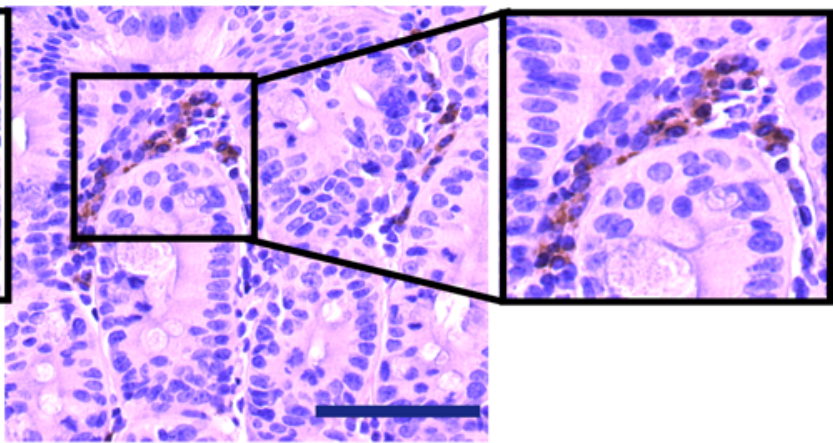

C
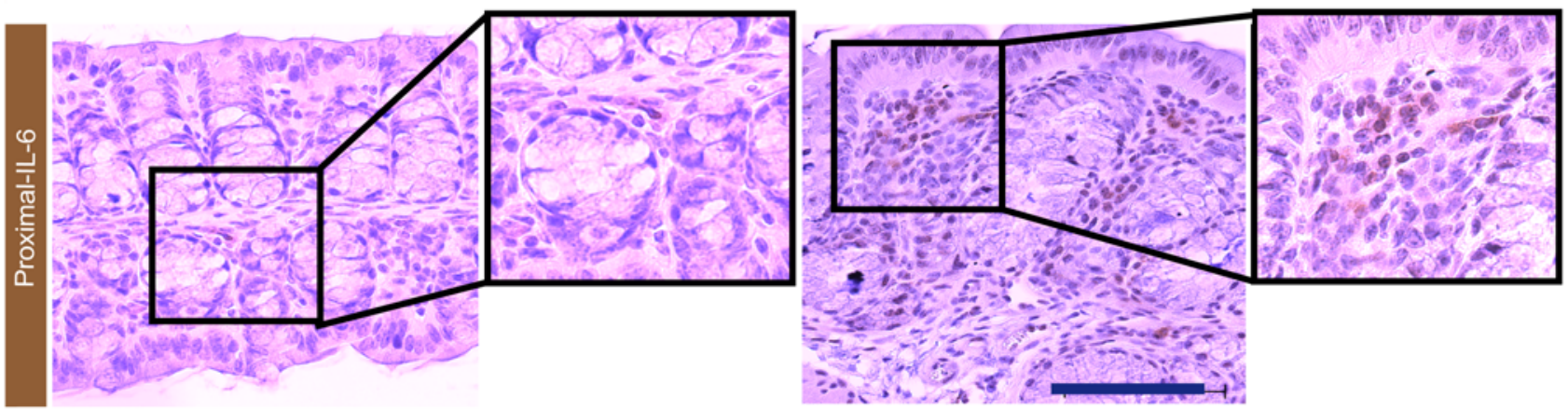

Figure 1. Cyclin A2 deletion leads to epithelial changes in the colon mucosa and inflammation. (A-C) Immunostaining for cyclin A2 (A), F4/80 (B), and IL-6 (C) of the indicated parts of colons from control (Ccna $\left.2^{f / f f}\right)$ and constitutive (VilCre Ccna2 $\left.2^{f / f f}\right)$ cyclin A2-deficient mice. Scale bars: $100 \mu \mathrm{m}$. Original magnification, $\times 2$ (enlarged insets).

We previously described a role for cyclin A2 in the regulation of cell migration and invasion (17-19). More specifically, we found that deletion of cyclin $\mathrm{A} 2$ in a murine mammary gland epithelial cell line (NMuMG) induces epithelial-mesenchymal transition (EMT) and increases invasiveness in vitro as well as in vivo in an avian embryo model $(18,19)$. This concurs with a recent report that identified cyclin $\mathrm{A} 2$ as a key regulatory component in EMT and metastasis of lung cancer cells by modulating the TGF- $\beta$ signaling pathway (20).

Few studies have investigated the role of cyclin A2 in tumor development in vivo using genetically altered mice. One study described the generation of mutant mice with reduced cyclin A2 expression. These mice displayed spontaneous tumor development, in particular in the lung, and were also more susceptible to 
chemically induced skin cancer (21). On the other hand, tissue-specific cyclin A2 inactivation in the liver was shown to impair hepatocellular carcinoma development and recapitulated the phenotype observed in absence of cyclin-dependent kinase 2 (CDK2) (22). These observations point to a tissue-specific role of cyclin A2.

We previously reported lower cyclin A2 expression in hepatic metastases compared with primary tumors derived from the same CRC patients (17). This prompted us to investigate the role of cyclin A2 in colon homeostasis and carcinogenesis using cell type-specific KO mice.

\section{Results}

Cyclin A2 depletion in the colon induces epithelial changes in the mucosa and inflammation. We established 2 mouse models to study the function of cyclin A2 in colon homeostasis and CRC development by using the previously established conditional cyclin A2 (Ccna $2^{f / f f}$ ) strain that is based on the Cre/LoxP system (23). Constitutive deletion of cyclin A2 in the intestinal epithelium was achieved by crossing cyclin A2 conditional-KO mice with the villin-Cre mouse strain (VilCre). Expression of Cre under the villin promoter was shown to be active in intestinal epithelial cells (24). In addition, we crossed $C c n a 2^{f / f l}$ mice with the inducible Cre mouse strain villin-CreERT2 (VilCreERT2), in which Ccna2 is excised upon intraperitoneal injection of tamoxifen (24).

We validated cyclin A2 loss in colonic crypts of VilCre Ccna2 $2^{f / f l}$ mice by immunohistochemical analysis (Figure 1A). For this, we analyzed the distal region, close to the anus, as well as the proximal region, close to the cecum (Supplemental Figure 1A; supplemental material available online with this article; https://doi.org/10.1172/ JCI131517DS1), since biological and clinical differences for both segments are well established (25). In both regions, we detected cyclin A2 expression in epithelial cells of the lower part of the crypts in control mice, whereas cyclin A2 expression was undetectable in colonic crypts of cyclin A2-KO mice (Figure 1A).

During the immunohistochemical analysis of cyclin A2 expression, we noted epithelial changes in the colonic crypts as well as architectural alterations in the mucosa of cyclin A2-deficient mice (Figure 1A and Supplemental Figure 1B). We also detected immune cell infiltration in cyclin A2-deficient colons. The immune cell infiltrates had morphological characteristics of macrophages and were immunostained using the macrophage marker F4/80. This revealed an increased number of $\mathrm{F} 4 / 80^{+}$cells, approximately 2-fold in constitutive (Figure 1B and Supplemental Figure 1C, left panel) and 1.5-fold in induced (Supplemental Figure 1C, right panel) cyclin A2-deficient colons versus normal ones. Macrophages can be important producers of the proinflammatory cytokine IL-6 (26), and, in fact, IL-6 was detectable in the stroma of cyclin A2-deficient colons but not in those of control mice (Figure 1C). Taking this one step further, we tested whether the infiltrating macrophages can be assigned to the M1 or M2 subtype, which have been attributed anti- and protumoral functions, respectively (27). Immunostainings for the M 2 markers YM1 and TGF- $\beta$ showed that colonic stroma of cyclin A2-deficient mice harbored predominantly M2-polarized macrophages (Supplemental Figure 1D).

Moreover, approximately $15 \%$ of male and $10 \%$ of female VilCre Ccna $2^{f / f l}$ mice developed rectal prolapses within the first 3 months of age, which can be considered an indicator of inflammation (28).
Taken together, cyclin A2 deficiency in colonic epithelial cells (CECs) induces epithelial and architectural alterations of colonic crypts as well as different signs of inflammation.

$R N A-S e q$ analysis reveals a prominent altered expression of genes involved in the cell cycle, the mitotic process, chromosome segregation, and DNA double-stranded break repair. To gain insights into the molecular traits of Ccna2-mutant mice, we performed RNA-Seq using CECs isolated from the proximal colon of Ccna2 $2^{f / f l}$ mice $(n=$ $4)$ and VilCre Ccna $2^{f / f l}$ mice $(n=4)$ at 4 weeks of age. At this age, VilCre Ccna $2^{f / f l}$ mice already displayed visible distortion of the crypt architecture that was validated by immunohistochemical analysis of the distal region of the colons used in this experiment.

Notably, the RNA-Seq experiment validated the deletion of exons 2 to 7 of the Ccna2 gene and thus the efficient inactivation of cyclin A2 in CECs of VilCre Ccna2 $2^{f / f l}$ mice (Supplemental Figure 2A). Supervised analysis of the RNA-Seq data identified 469 upregulated and 640 downregulated genes in VilCre Ccna2 $2^{f / f l}$ mouse samples versus $C c n a 2^{f / / f l}$ mouse samples (fold change $>2$, adjusted $P$ value $|\mathrm{FDR}|>0.05$ ) (Supplemental Table 1). Pathway analyses of the most altered genes revealed that the most significant biological functions associated with the genes upregulated in VilCre Ccna2 $2^{f / f l}$ mice were related to the cell cycle (nuclear division and mitosis, regulation of the mitotic cell cycle, $P<2.79 \times 10^{-3}$ to $1.15 \times 10^{-12}$ ), cellular assembly and organization (chromosome segregation, chromatin segregation, $P<3.01 \times 10^{-3}$ to $1.80 \times 10^{-9}$ ), as well as DNA replication, recombination, and repair (double-stranded break repair [DSB] and homologous recombination, $P<3.01 \times 10^{-3}$ to 1.80 $\times 10^{-9}$ ) (Supplemental Tables 1 and 2 and Supplemental Figure 2B). In line with this, the analysis highlighted increased levels of Mki67 (coding for Ki67), Brca1, Brca2, Rad18, Rad51, Blm, Exo1, or Rpa1 (genes involved in the DSB repair mechanism) as well as $C d c 45$, Bub1, Mad2, and Rad21 (genes related to checkpoint controls of the cell cycle and to abnormal mitosis) (Supplemental Figure 2C).

Cyclin A2 loss in CECs leads to increased proliferation of CECs and onset of dysplasia. To validate the RNA-Seq results described above at the functional level, we first tested the proliferation status of cyclin A2-deficient CECs by measuring BrdU uptake. For this, mice received an intraperitoneal injection of the nucleoside analog BrdU and were sacrificed 2 hours later to isolate colons. We performed subsequent immunohistochemical analysis by dissecting the different regions of the colon as described above (Supplemental Figure 1A) and by separating the bottom, middle, and top parts of the crypts. As shown in Figure $2 \mathrm{~A}, \mathrm{BrdU}^{+}$cells were mainly located in the bottom of the crypts in control mice. In contrast, BrdU incorporation was most prominent in the middle part of the cyclin A2-deficient crypts, with an average of more than 4 cells per crypt $(n=60, P<0.0001)$. Moreover, we detected BrdU ${ }^{+}$cells in the upper crypts of VilCre Ccna $2^{f / f l}$ mice but not in those of control mice. These specific profiles of BrdU incorporation of cyclin A2-deficient CECs were observed equally in the distal, transverse, and proximal regions. To confirm the increased proliferation rate of cyclin A2-deficient CECs, we analyzed Ki67, which is expressed during all active phases of the cell cycle (i.e., $G_{1}, S, G_{2}$, and $M$ ) but is absent in resting cells (29). We detected Ki67 expression in the distal region of colons at the bottom of the crypts in colons of control mice, but its expression was localized to the middle and upper parts of the colonic crypts of Ccna2-KO animals. We observed a 


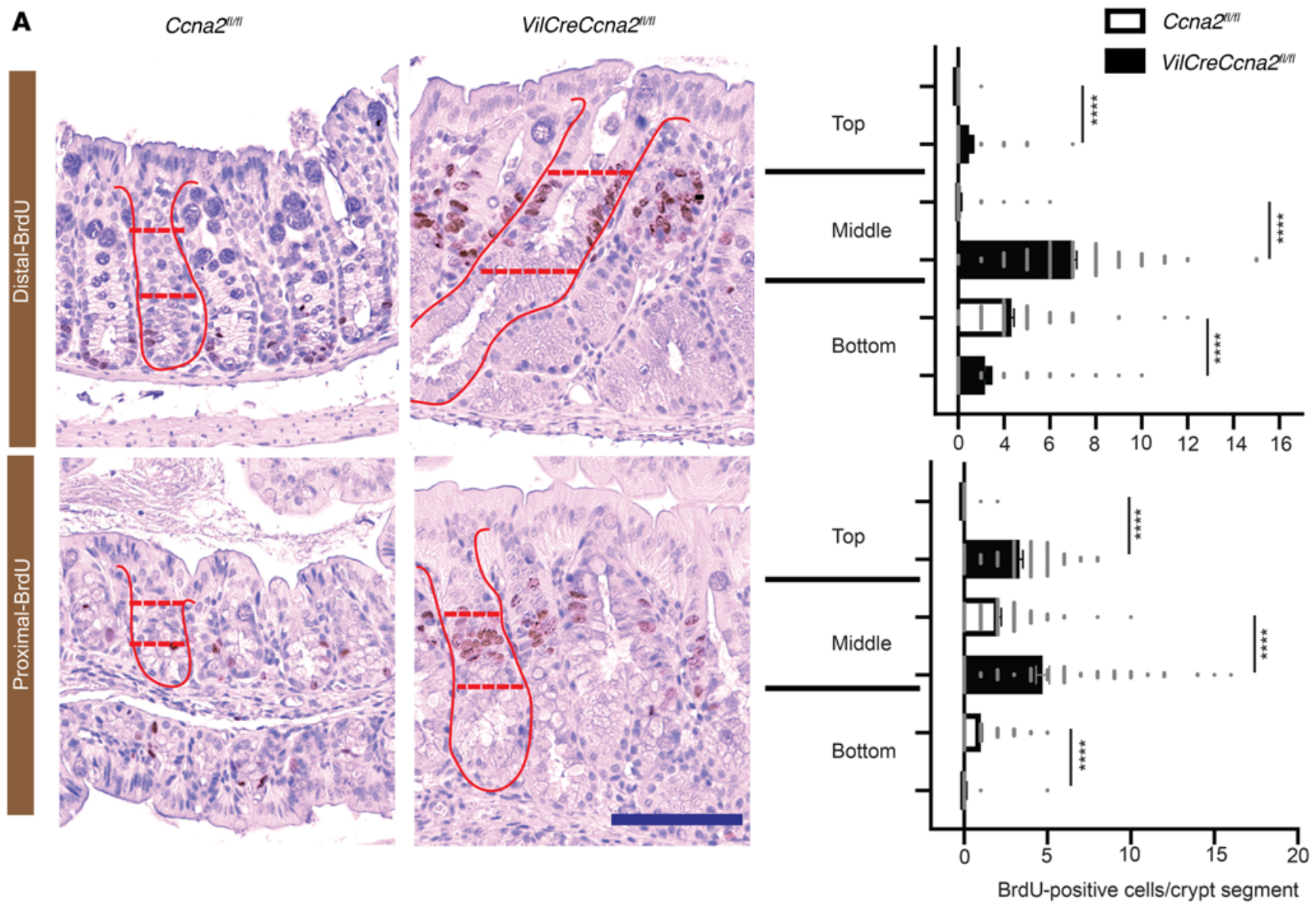

B Ccna2 $2^{\text {unt }}$ VilCreERT2Ccna2 $2^{\text {wn }}$

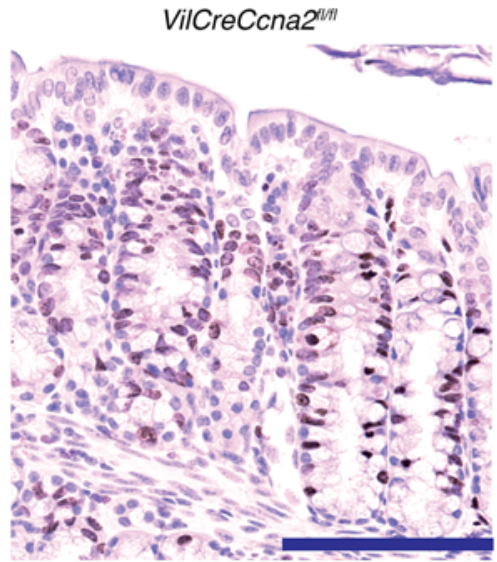

Figure 2. Cyclin A2 depletion in CECs promotes cell proliferation. (A) Analysis of cell proliferation in colonic crypts of control (Ccna2 $\left.2^{f / f f}\right)$ and cyclin A2deficient (VilCre Ccna2 $\left.{ }^{f / / f l}\right)$ mice by BrdU incorporation. Shown are representative images of immunostainings for BrdU of the distal and proximal parts of colons taken from Ccna2 $2^{f / f l}$ and VilCre $C \mathrm{cna}^{f / / f l}$ mice 2 hours after intraperitoneal injection of the nucleoside analog. Plots show quantification of the number of $\mathrm{BrdU}^{+}$cells per crypt segment. Crypts (outlined in red in the images) were subdivided into 3 parts (dotted lines) for the analysis (bottom, middle, and top: $n=120$ crypts for the distal and $n=110$ crypts for the proximal parts of colons from 3 different mice). Data represent the mean \pm SEM. ${ }^{* * * *} P<0.0001$, by unpaired, 2-tailed Student's $t$ test. (B) Representative images of Ki67 immunostaining in the proximal part of colons from control animals (Ccna ${ }^{\left.f^{f / f f}\right)}$, induced KO mice 8 days after tamoxifen treatment (VilCreERT2 (cna2 $\left.2^{f / f l}\right)$, and constitutive KO mice (VilCre Ccna2 $\left.2^{f / f l}\right)$. Scale bars: $100 \mu \mathrm{m}$.

similar pattern in the proximal region of colons (Figure 2B and Supplemental Figure 3, A and B) from constitutive as well as induced KO mice (Supplemental Figure 3C), therefore confirming the observation made in the BrdU-uptake experiment. Thus, the elevated expression levels of Ki67 observed by immunostaining in cyclin A2-deficient colons concur with the upregulation of Mki67 transcripts detected in the RNA-Seq analysis.

We next tested whether expansion of the proliferative compartment in the middle part of cyclin A2-deficient crypts is associated with reprograming toward a stem cell-like phenotype. For 
this, we used CD44v6, which was originally described as a marker for constitutive and reprogrammed cancer stem cells (30) but was later found to also be expressed by cells at the bottom of normal colonic crypts, where colonic epithelial stem cells are localized (31). Indeed, we detected distinct expression of CD44v6 by cells at the base of the normal crypts, but a strong upregulation of CD44v6 levels in the abnormal Ki67 ${ }^{+}$compartment of cyclin A2deficient crypts (Supplemental Figure 4).

Strikingly, in approximately $80 \%$ of the mice analyzed, colons deficient for cyclin A2, either constitutive or induced, revealed low- and high-grade dysplasia that was mostly found in the proximal region (Figure 3, A and $\mathrm{B}$ ). Constitutive $\mathrm{KO}$ mice showed an average occurrence of 4 low-grade dysplasias and nearly 2 high-grade dysplasias per colon. Induced KO mice had a mean of approximately 3 low-grade and high-grade dysplasias per colon, whereas no dysplasia was detectable in the control mice (Figure 3, C and D). Notably, dysplasia was already detectable 8 days after tamoxifen injection in VilCreERT2 Ccna $2^{f l / f l}$ mice.

Cyclin A2 depletion in CECs leads to abnormal mitoses and DNA damage. We further explored cell-cycle features and noticed an increased incidence of mitoses within the crypts when analyzing colons of constitutive and induced $\mathrm{KO}$ mice (on day 8). Colons with constitutive and induced Ccna2 deletion showed an average of 13 and 24 mitotic figures per $\mathrm{mm}^{2}$, respectively, whereas in control animals, we observed mitotic figures that were on average only 2 per $\mathrm{mm}^{2}$ ( $n=5, P<0.01$ for the constitutive as well as induced KO mouse strain) (Figure 4, A and B). In addition, CECs deficient for cyclin A2 harbored nuclear pleomorphisms characterized by increased nuclear size compared with controls (Figure 4A and Supplemental Figure 5). Finally, abnormal mitosis, detected by combined staining for $\alpha$-tubulin to visualize mitotic spindles, $\gamma$-tubulin to label centrosomes, and DAPI to identify chromosomes, was prominent in induced cyclin A2-deficient colons but not in control colons (Supplemental Figure 6, A and B).

Defective mitoses can lead to DNA damage (32), thus, we analyzed colons of tamoxifen-treated VilCreERT2 Ccna2 $2^{f / f l}$ mice by IHC using a marker for DSBs, i.e., an anti- $\gamma \mathrm{H} 2 \mathrm{Ax}$ antibody, and found an average of more than $70 \gamma \mathrm{H} 2 \mathrm{AX}^{+}$cells $/ \mathrm{mm}^{2}$ in cyclin A2deficient CECs compared with 40 in control CECs (Figure 4C; $n=$ $4, P<0.05)$. Mre11 is part of the NMR complex involved in the resolution of dsDNA (33). We therefore analyzed Mre11 foci formation by confocal microscopy (34) and found that Mre11 foci formation was significantly increased in VilCre Ccna2 $2^{f / f l}$ nuclei (Figure 4D).

Altogether, the alterations found at the protein level correlated with the RNA-Seq results, underpinning the presence of cell-cycle perturbations, increased DNA damage, and DNA DSB repair pathway activation in cyclin A2-deficient CECs.

Activation of regenerative pathways in cyclin A2-deficient colons. Tissue injury induces inflammation and, in turn, a regenerative response (35). Several pathways have been described that can promote tissue repair in the intestine including YAP/TAZ, NF- $\kappa \mathrm{B}$, and WNT/ $\beta$-catenin signaling $(35,36)$. We have previously shown that cyclin A2 inactivation in epithelial cells in vitro leads to increased $\beta$-catenin stability and activity $(18,19)$. For this reason, we first tested $\beta$-catenin expression in the colons of constitutive $\mathrm{KO}$ mice by immunohistochemical analysis, which revealed increased nuclear localization of $\beta$-catenin in CECs of cyclin A2-deficient colons (Figure $5 \mathrm{~A}$ ).
In addition, Western blot analysis of nuclear extracts derived from the constitutive $\mathrm{KO}$ mice displayed elevated levels of both active $\beta$-catenin and the NF- $\kappa \mathrm{B}$ p65 subunit (Figure 5B, left panel). We also detected TAZ expression in crypts as well as surrounding stroma in colons of VilCre Ccna2fl/fl mice (Figure 5D, right panel).

To investigate the activation sequence of the different pathways modulated in cyclin A2-deficient CECs at the protein level, we took advantage of the inducible Cre mouse model (VilCreERT2 Ccna2 $2^{f / f l}$ mice). Two tamoxifen injections on days 0 and 2 were sufficient to abrogate cyclin A2 expression in VilCreERT2 Ccna $2^{f / f l}$ mice (Figure $5 \mathrm{~B}$, middle and right panels). We performed Western blot analysis on days 2 and 4 upon Ccna2 depletion when the colons still showed no alterations such as changes in crypt architecture or immune cell infiltration (Supplemental Figure 7, A and B). We measured the expression levels of active $\beta$-catenin and NF- $\kappa \mathrm{B}$ by Western blotting (Figure 5B), as well as expression of $\gamma \mathrm{H} 2 \mathrm{AX}$ and TAZ by IHC (Figure 5, C and D, and Supplemental Figure 7A). In addition, we performed quantification of mitoses on $\mathrm{H} \& \mathrm{E}$-stained colons from induced KO mice, also on days 2 and 4 (Supplemental Figure 7B, right panel). An increase in mitoses as well as DNA damage, monitored by $\gamma \mathrm{H} 2 \mathrm{AX}$ staining, were already detectable on day 2 and were further increased on day 4 in cyclin A2-deficient CECs (Figure 5C, and Supplemental Figure 7, A and B). This concurs with the elevated number of Mre11 foci, which were detectable by day 2 on and progressed with time after cyclin A2 inactivation (Supplemental Figure 7C). In contrast, activation of $\beta$-catenin was only detectable on day 4 , thus after the induction of DNA damage on day 2, whereas $\mathrm{NF}-\kappa \mathrm{B}$ and TAZ levels were unaltered at those time points (Figure $5 \mathrm{~B}$, middle and right panels, and Figure 5D). To test whether elevated $\beta$-catenin activation (nuclear staining) and/or DNA damage are associated with the abnormal $\mathrm{Ki}^{+}{ }^{+}$compartment in cyclin A2-deficient colons, we performed costainings, which revealed that nuclear active $\beta$-catenin and $\gamma \mathrm{H} 2 \mathrm{AX}$ were predominantly present in the lower and upper parts of the crypts, respectively, and that these parts were devoid of Ki67 (Supplemental Figures 8 and 9).

To further explore the alterations in cyclin A2-deficient colonic epithelium, we generated organoids. During the process of extraction from colon, we noticed an increased fragility of the cyclin A2-deficient crypts, yielding a reduced number of crypts when compared with controls, with a mean of 2300 and 9500 crypts per colon, respectively ( $n=7, P<0.001$; Figure 6A). More important, while colon organoids derived from $C c n a 2^{f / f l}$ mice were budding on day 4 of culturing, the organoids derived from VilCre $C c n a 2^{f / f l}$ mice were mainly forming cysts in the presence of normal organoid media containing WNT3a (i.e., WENR media containing WNT3a, EGF, noggin, R-spondin) (Figure 6B). Cyst formation has been observed on intestinal organoids from APC-mutant mice (37). We therefore cultivated cyclin A2-deficient crypts in the absence of WNT3a (ENR media) and found them to be able to bud under this condition (Figure 6C). The formation of cysts in cyclin A2-deficient organoids appeared therefore be related to an increased activation of $\beta$-catenin signaling, thus concurring with the results obtained by Western blotting and immunohistological analysis of colons.

Cyclin A2 deficiency in CECs promotes colon carcinogenesis in mice. To test whether cyclin A2 deficiency modulates colon carcinogenesis, we subjected VilCre Ccna2 $2^{f / f l}$ and Ccna2 $2^{f / f l}$ mice to chemically induced colon carcinogenesis, an established mouse 
A VilCreCcna2

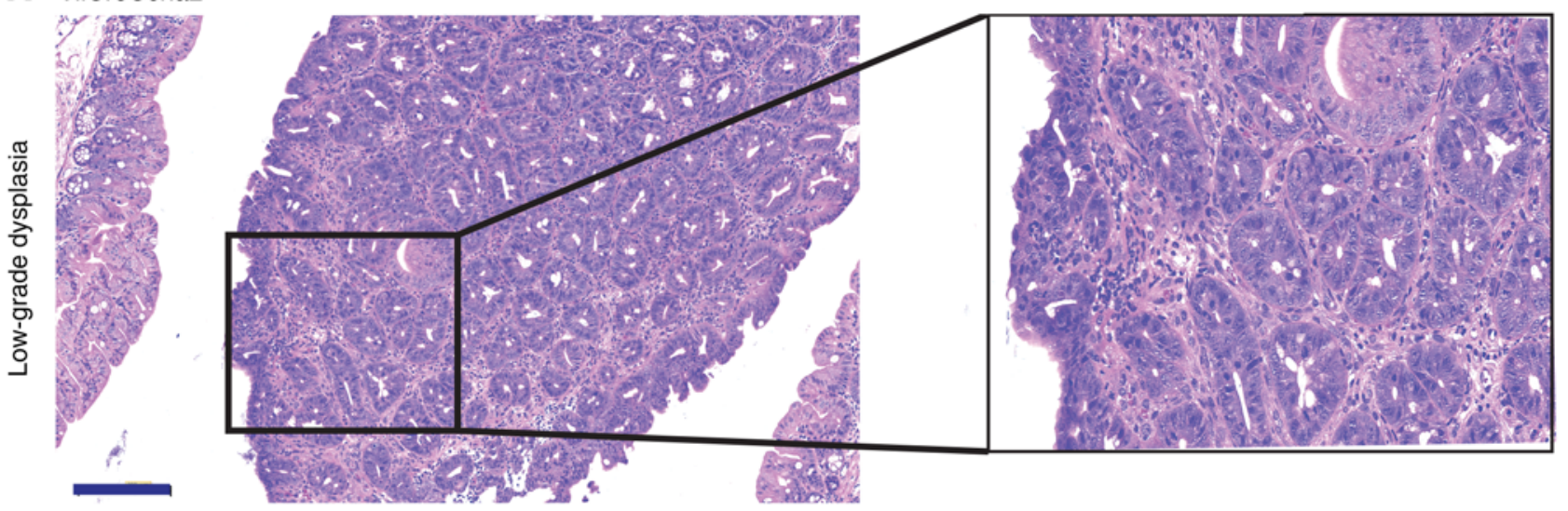

B VilCreERT2Ccna2

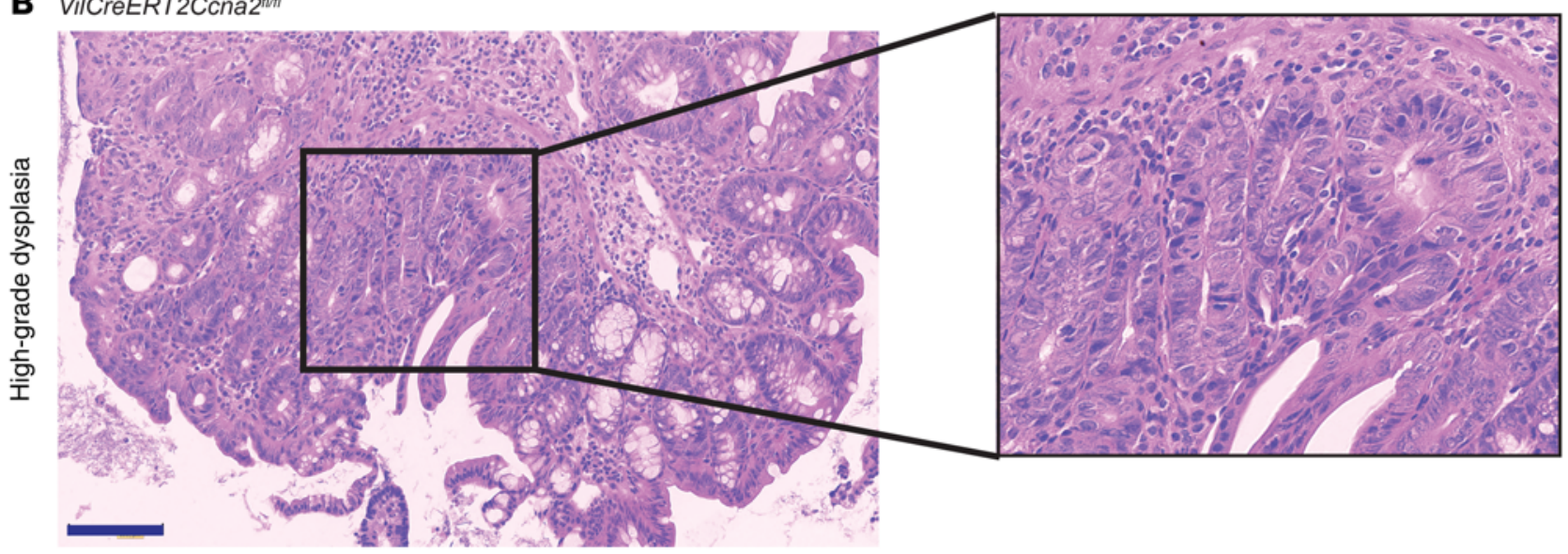

C
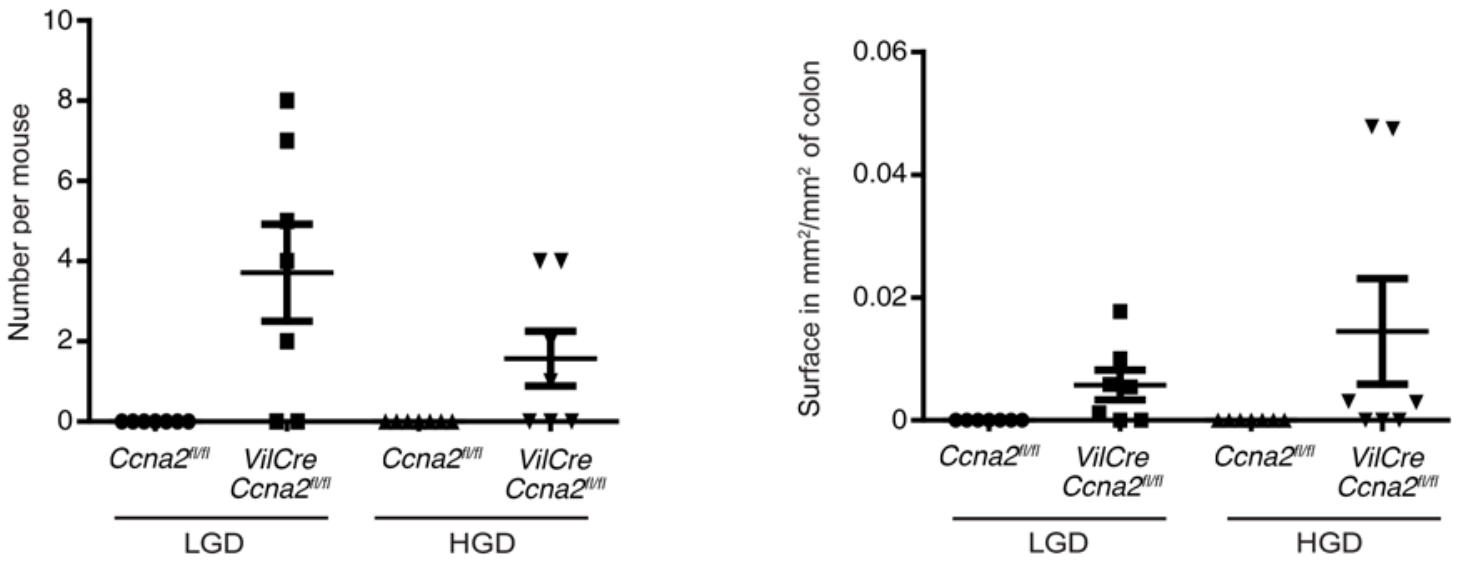

D
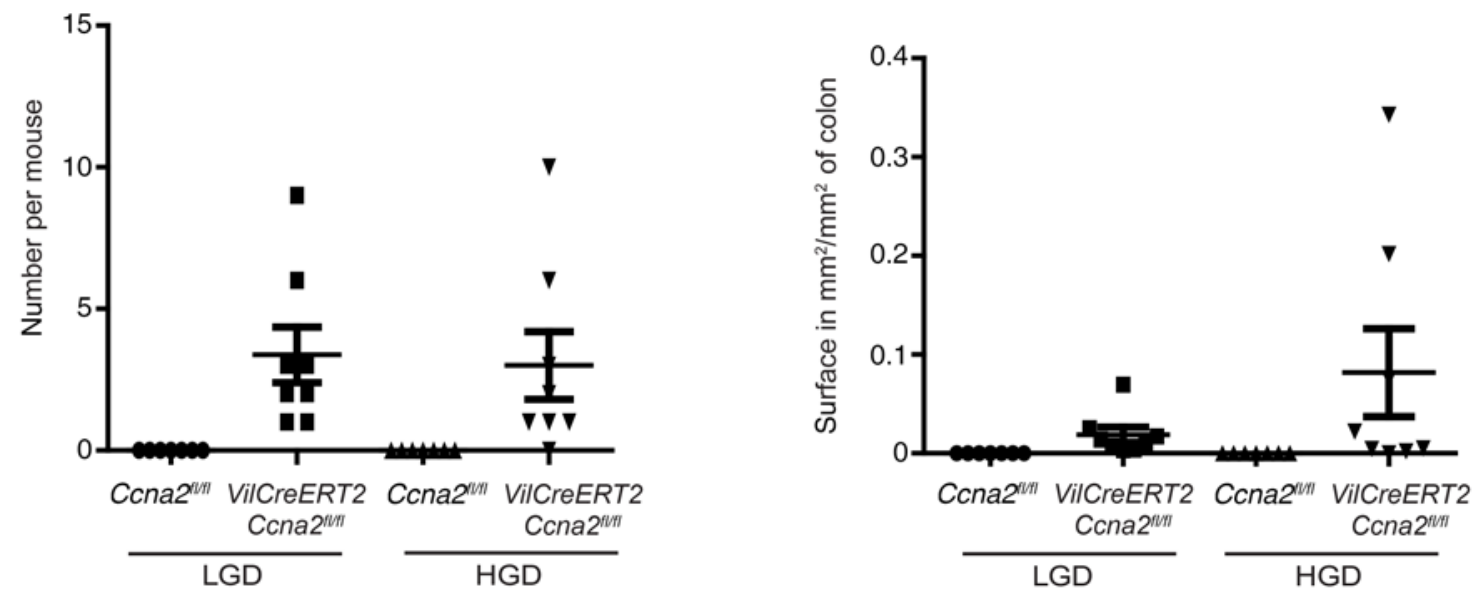
Figure 3. Dysplasia formation in cyclin A2-deficient mice. (A and B) Representative images of $\mathrm{H} \& \mathrm{E}$ staining showing low-grade dysplasia (LCD) and high-grade dysplasia (HCD) in the colons of constitutive (VilCre Ccna2 $\left.2^{f / f 1}\right)(\mathbf{A})$ and tamoxifen-induced (VilCreERT2 CCna2 ${ }^{f / f}$ ) KO (B) mice. Scale bars: 100 $\mu \mathrm{m}$. Original magnification, $\times 2$ (enlarged insets). (C and $\mathbf{D})$ Quantification of the number of low- and high-grade dysplasias and dysplasia areas (in $\mathrm{mm}^{2} /$ $\mathrm{mm}^{2}$ of colon) in constitutive VilCre $\mathrm{Ccna}^{f / / f l}(n=7)$ mice and VilCreERT2 Ccna $2^{f / f l}$ induced KO mice 8 days after tamoxifen treatment $(n=8)$ versus control $C \mathrm{Cna} 2^{f l / f l}$ animals $(n=7)$. Data represent the mean \pm SEM. Statistical analysis was performed using an unpaired, 2-tailed Student's $t$ test.

model resembling the human pathology (38). This model depends on the intraperitoneal injection of the mutagen azoxymethane $(\mathrm{AOM})$ and the subsequent induction of inflammation by adding dextran sodium sulfate (DSS) in the drinking water, and is therefore named colitis-associated carcinogenesis (CAC). DSS is toxic to mucosal epithelial cells, specifically in the colon, and the destruction of the mucosal barrier leads to chronic inflammation. In the classical protocol, mice receive 3 DSS treatments for 5 days within 60 days, leading to tumor lesions in the distal region of the colon (38). VilCre C $\mathrm{Cna} 2^{f / / l}$ mice displayed an approximately 2-fold greater weight loss on days 10 through 15 following the first DSS administration compared with control mice (Supplemental Figure 10). We therefore decided not to administer additional doses of DSS to the mice. Mice were sacrificed 60 days after the AOM injection, and colons isolated and embedded in paraffin. The grade of colitis was evaluated as described in Supplemental Table 3 (39) and was 3 -fold higher in cyclin A2-deficient mice $(n=6, P \leq 0.05$; Figure $7 \mathrm{~A})$. Differences included submucosal alterations as well as transmural disruption by immune cell infiltration, which was undetectable in control mice (Figure 7, A and B). The colon architecture was strongly affected in cyclin A2-deficient mice (Figure 7C), and the incidence of low- and high-grade dysplasia was pronounced in the VilCre Ccna $2^{f / / l}$ mice but was rather rare in the control animals (Figure 7D). Moreover, the surface area of both low- and high-grade dysplasias was significantly higher in the cyclin A2-deficient mice compared with the control animals (Figure 7E). In addition, adenocarcinomas were only detectable in cyclin A2-deleted mice, but not in controls (Figure 7, D and E). Immunohistochemical analysis revealed increased expression levels of activated $\beta$-catenin, $\gamma \mathrm{H} 2 \mathrm{AX}$, as well as IL-6 in cyclin A2-deficient colons compared with levels in the control animals (Figure 8).

We next evaluated the impact of cyclin A2 deletion on established tumor lesions. For this, we exposed VilCreERT2 Ccna $2^{\text {f/fl }}$ and $C c n a 2^{f / f l}$ mice to the classical AOM/DSS protocol described in Figure 9A and subsequently induced cyclin A2 deletion by 2 consecutive tamoxifen injections. Approximately 40 days later, the mice were sacrificed for immunohistological analysis that revealed an increased number of adenocarcinomas in cyclin A2deficient colons (Figure 9B), which were characterized by elevated expression levels of the stemness marker CD44v6 (30) (Figure 9, $\mathrm{C}$ and D). Strikingly, invasive adenocarcinomas were detectable only in the proximal part of the colons of cyclin A2-deficient mice and absent in those of controls. Notably, the invasive adenocarcinoma displayed abundant CD44v6 expression, whereas the desmoplastic stroma of the invading tumors showed prominent TGF- $\beta$ expression (Figure 9C).
Altogether, these observations show that cyclin A2 deficiency in CECs promoted chemically induced colon carcinogenesis in mice.

Cyclin A2 expression in patients with CRC has prognostic value. Our finding that cyclin A2 deficiency in mice promoted spontaneous development of dysplasia in the colon as well as induced colon carcinogenesis prompted us to perform a meta-analysis of CCNA2 expression on available public data sets (Supplemental Table 4). The characteristics of the 2239 patients with CRC profiled are summarized in Supplemental Table 5. Briefly, the median age of the patients was 68 years (range, 19-97 years), and the sex ratio was balanced, with $47 \%$ of female patients and $53 \%$ male patients. The most frequent location was the right colon, followed by the left colon and sigmoid. The pathological stage included more stage 3-4 than stage 1-2 cancers. The pathological grade was mostly 2 , and the mismatch repair (MMR) status was microsatellite stable (MSS) in most cases. The CMS classification identified 20\% of samples as CMS1 (microsatellite instability [MSI] immune), 32\% as CMS2 (canonical), 17\% as CMS3 (metabolic), and 31\% as CMS4 (mesenchymal).

CCNA2 expression was heterogeneous across the 2239 primary CRC (pCRC) tumors analyzed (Figure 10A). Compared with normal colon (NC) tissues, CCNA2 transcript levels were upregulated in most of the primary tumors. In contrast, expression levels in CRC metastases (ANOVA, $P=4.16 \times 10^{-54}$ ) were significantly lower compared with levels in primary tumors (Figure 10A). In addition, we found that CCNA2 transcript levels were lower in the stages 3-4 versus stages 1-2 (Figure 10B). In parallel, we used 2 different cyclin A2 antibodies to evaluate cyclin $\mathrm{A} 2$ protein expression by IHC on a tissue microarray (TMA) of CRC biopsies taken from 65 patients from stage 1 to stage 4 (described in Supplemental Table 6) (Figure 10C, and Supplemental Figure 12A, and see Methods). We quantified cyclin A2 expression as the number of epithelium-positive cells per $\mathrm{mm}^{2}$ of tumor area. Immunohistochemical analysis confirmed the transcriptome data by revealing cyclin A2 protein levels to be lower in stages 3 and 4 than in early stages (Figure 10C, and Supplemental Figure 12A). In addition, we observed lower cyclin A2 levels in stage 2 MSS compared with levels detected in MSI tumors (Figure 10C, and Supplemental Figure 12, A and B). We next evaluated CCNA2 levels according to the CMS classification (8) and observed that CCNA2 expression was highest in the CMS1 class, lowest in the CMS4 class, and intermediate in the CMS2 and CMS3 classes (Figure 10E).

We next measured CCNA2 expression as a discrete value after comparison with the median expression of the $95 \mathrm{NC}$ samples; upregulation, hereafter designated as $C C N A 2^{\mathrm{hi}}(n=1139)$, was defined as a CRC/NC ratio of 2 or higher, and no upregulation, designated as CCNA $2^{\text {non-hi }}(n=1100)$, was defined as a CRC/NC ratio of less than 2. We searched for correlations between the CCNA $2^{\text {hi }}$ or CCNA2 $2^{\text {non-hi }}$ classification and the clinicopathological and molecular variables of the $2239 \mathrm{pCRC}$ samples (Supplemental Table 5) and found no correlation between CCNA2 mRNA levels and patients' age or sex. By contrast, when compared with the CCNA2 $2^{\text {non-hi }}$ class, the CCNA $2^{\text {hi }}$ class was associated with more frequent right and left tumor locations $\left(P=2.04 \times 10^{-2}\right.$, Fisher's exact test), pathological stages 1 and $2\left(P=3.19 \times 10^{-8}\right.$; Supplemental Table 5 and Figure 10B), pathological grade $3\left(P=5.99 \times 10^{-03}\right)$, as well as MSI status $(P$ $\left.=1.48 \times 10^{-5}\right)$ and CMS1 molecular subtype $\left(P=1.00 \times 10^{-6}\right)$.

Relapse-free-survival (RFS) data were available for 1882 of 2239 operated pCRC samples. The median follow-up was 42 

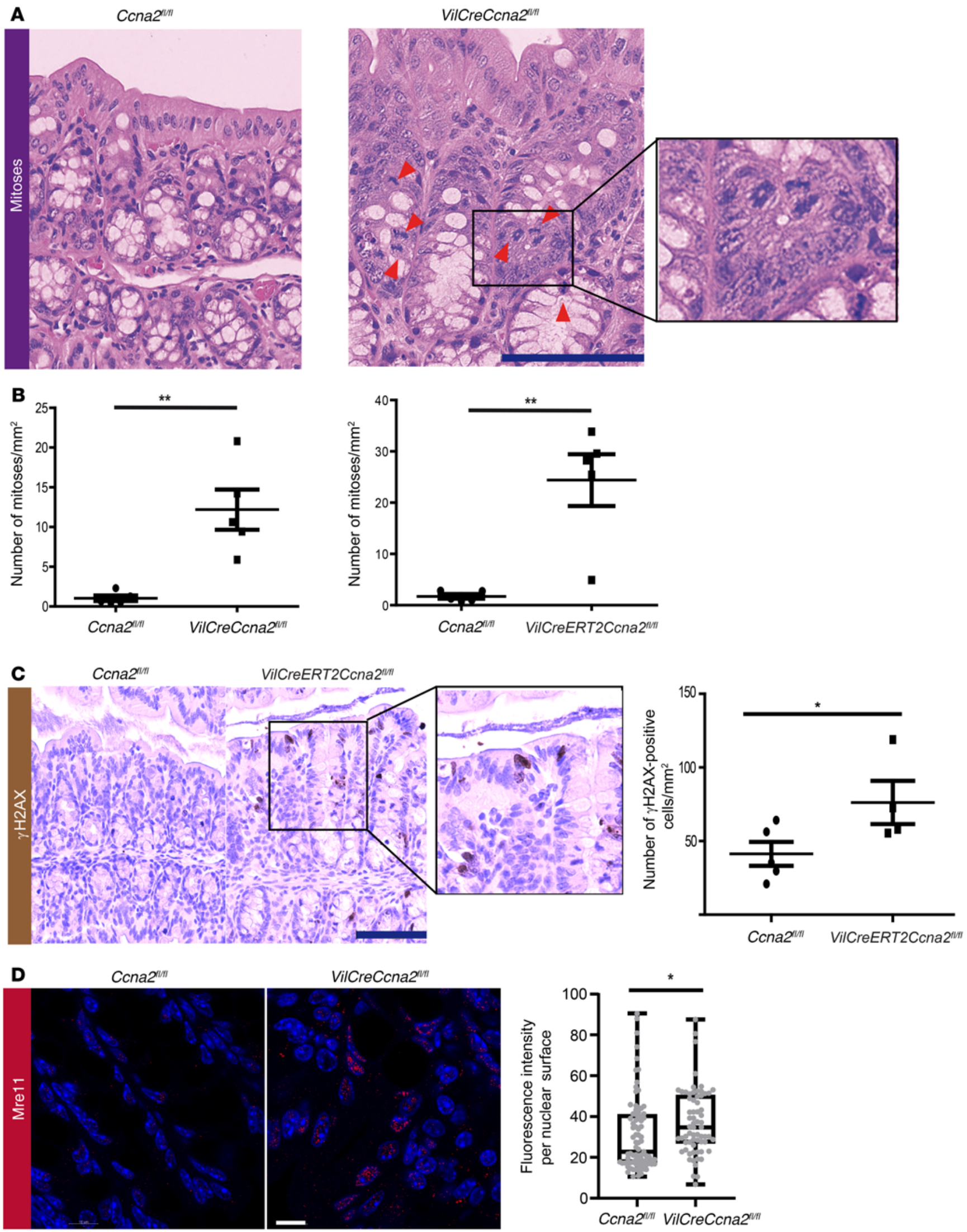
Figure 4. Elevated mitoses and DNA damage following cyclin A2 deletion in the colonic epithelium. (A) Representative H\&E staining showing an increased number of mitoses (red arrowheads) in the proximal region of colon from a cyclin A2-deficient mouse compared with colon from a control animal. Scale bar: $100 \mu \mathrm{m}$. Original magnification, $\times 2$ (enlarged inset). (B) Quantification of mitotic events in constitutive mice (left: $n=5$ ), induced KO mice 8 days after cyclin A2 inactivation (right: $n=5$ ), and control animals $(n=5)$. Data represent the mean \pm SEM. ${ }^{* *} P<0.01$, by unpaired, 2 -tailed Student's $t$ test. (C) Immunohistochemical analysis of $\gamma \mathrm{H} 2 \mathrm{AX}$ expression in colon sections of control Ccna2 $2^{f / f l}$ mice $(n=5)$ and VilCreERT2 Ccna2 fl/fl $^{\prime \prime l}$ tamoxifen-induced KO mice $(n=4)$ on day 8 following cyclin A2 inactivation. Representative $\gamma \mathrm{H} 2 \mathrm{AX}$ immunostaining images and quantification of the number of $\gamma \mathrm{H}_{2} \mathrm{AX}+$ cells per $\mathrm{mm}^{2}$ of epithelium from the proximal part of the colon are shown. Data represent the mean \pm SEM. ${ }^{*} P<0.05$, by unpaired, 2-tailed Student's $t$ test. Scale bar: $100 \mu \mathrm{m}$. Original magnification, $\times 2$ (enlarged inset). (D) Confocal analysis of nuclear foci formation of Mre11 in crypts from the proximal part of colons from control Ccna2 $2^{f / f l}$ and VilCre $\mathrm{CCna}^{\mathrm{fl} / \mathrm{fl}}$ mice. Representative images are shown. Nuclei are stained with DAPI (blue) and Mre11 (red). Scale bar: $10 \mu \mathrm{m}$. Plot shows quantification of Mre11 fluorescence intensity per nuclear surface ( $n=83$ different nuclear areas for control mice and $n=65$ for cyclin A2-deficient mice). Data represent the mean \pm SEM. ${ }^{*} P<0.05$, by unpaired, 2 -tailed Student's $t$ test.

months (range, 1-212); 496 patients (26\%) had an event, and the 5-year RFS was 70\% (95\% CI: 68-73; Supplemental Figure 11A). Interestingly, the clinical outcome was different between the two CCNA2-based classes, with 281 events $(31 \%)$ in the CCNA2 $2^{\text {non-hi }}$ class $(n=894)$ versus 215 events $(22 \%)$ in the CCNA2 ${ }^{\text {hi }}$ class $(n=$ 988; $P=2.32 \times 10^{6}$, Fisher's exact test; Supplemental Table 5). The 5-year RFS was lower (65\%) for CCNA2 $2^{\text {non-hi }}$ patients $(95 \%$ CI: 61-69) versus 75\% (95\% CI: 72-79) for the CCNA2 $2^{\text {hi }}$ patients, respectively $\left(P=8.86 \times 10^{-6} ;\right.$ Figure $\left.10 D\right)$. In univariate analysis for RFS (Supplemental Table 7), the HR for the event was 0.67 (95\% CI: $0.56-0.80)$ in the CCNA2 $2^{\text {hi }}$ class when compared with the HR for the CCNA2 $2^{\text {non-hi }}$ class $\left(P=1.01 \times 10^{-5}\right.$, Wald's test). Other variables associated with RFS included the tumor location $(P=1.35 \times$ $\left.10^{-6}\right)$, the pathological stage $\left(P=2.78 \times 10^{-15}\right)$, and MMR status $(P=$ $2.97 \times 10^{-4}$ ) (with the CCNA2 $2^{\text {non-hi }}$ class being predominantly associated with MSS status, in agreement with the results obtained with the TMA), unlike patients' age, sex, and pathological grade. In multivariate analysis (Supplemental Table 7), the CCNA2-based classification remained associated with RFS ( $P=2.99 \times 10^{-3}$, Wald's test), as well as tumor location (right and sigmoid colon), pathological stage, and MMR status. Of note, this association remained significant $\left(P=4.13 \times 10^{-4}\right)$ in multivariate analysis incorporating the CMS molecular classification (Supplemental Table 7). As shown in Figure $10 F$, the RFS was affected for the 8 classes defined by both CCNA2 expression levels and CMS $\left(P=1.82 \times 10^{-6}, \log\right.$-rank test): CCNA2 expression levels correlated with the clinical outcome for $3 \mathrm{CMSs}$, with a significantly reduced RFS for patients with CCNA2 $2^{\text {non-hi }}$ tumors compared with patients in the CCNA2 ${ }^{\text {hi }}$ class for CMS1 (59\% versus $\left.73, P=1.64 \times 10^{-2}\right)$, CMS3 (62\% versus $\left.79 \%, P=0.05\right)$, and CMS4 subtypes ( $60 \%$ vs. $\left.74 \%, P=2.08 \times 10^{-2}\right)$ (Figure $10 \mathrm{E}$ ).

To further explore the biological alterations associated with CCNA2 expression, we compared the whole-genome expression profiles of CCNA2 $2^{\text {non-hi }}(n=222)$ and $C C N A 2^{\text {hi }}(n=237)$ The Cancer Genome Atlas (TCGA) samples (learning set) (Supplemental Figure 11,B and C). We identified 92 genes that were differentially expressed, including 20 genes overexpressed and 72 genes underexpressed in the CCNA2 $2^{\text {hi }}$ class (Supplemental Figure 11C, and Supplemental Table 8). We used the gene expression signature (GES) of 92 genes based on the metagene analysis to classify samples. As expected, this GES allowed efficient classification of the 459 learning set samples ( $P=1.08 \times 10^{-52}$, Student's $t$ test). More important, we validated its robustness in a completely independent validation set that included the 1780 remaining samples $\left(P=9.22 \times 10^{-180}\right.$, Student's $t$ test; Supplemental Figure 11B). A detailed analysis of the 92 genes revealed that the 20 most significantly upregulated genes are involved in cell proliferation and DNA repair (mitotic cell-cycle regulation, genomic instability, and replicative immortality pathways) (Supplemental Table 8).

\section{Discussion}

Cyclins are well-established drivers of the cell cycle through activation of a specific family of kinases, the cyclin-dependent kinases (CDKs). Gene amplification as well as overexpression of cyclins have been frequently detected in cancer tissues, and cyclins have been proposed as biomarkers or therapeutic targets (40). For example, inhibition of cyclin A2 complexes has been shown to impair the proliferation of some tumor cell lines (41).

Here, we report that cyclin A2 deficiency in CECs led to epithelial changes in the mucosa, induced inflammation, and increased cell proliferation and dysplasia in the colon, rendering the mice more susceptible to chemically induced colon carcinogenesis. A higher incidence of mitoses as well as DNA DSBs were detectable as early as 2 days after cyclin A2 inactivation and appeared, therefore, to be the initial events, as elevated activation of $\beta$-catenin was only detectable on day 4, upon cyclin A2 deletion, followed later on by activation of NF- $\mathrm{BB}$ and TAZ. Therefore, cyclin A2 deficiency in CECs led to different molecular alterations, with DNA damage and abundant mitoses as the initiating events, followed by activation of $\beta$-catenin and the regenerative and inflammatory pathways involving TAZ and NF-кB. Our immunohistochemical analysis suggested a spatial separation of DNA damage and $\beta$-catenin activation in cyclin A2-deficient colons, with nuclear staining of active $\beta$-catenin being more frequent in the lower part of the colonic crypts, i.e., below the proliferative $\mathrm{Ki} 7^{+}$compartment, whereas $\gamma \mathrm{H} 2 \mathrm{AX}$ staining was localized in the upper crypt region (Supplemental Figures 8 and 9). Furthermore, we found that the expanded proliferative $\mathrm{Ki}^{+} 7^{+}$compartment in cyclin A2-deficient colons gained a stem-like phenotype as revealed by immunostaining for CD44v6, an established colon cancer stem cell marker and driver of metastasis (30).

It is well established that the WNT/ $\beta$-catenin pathway triggers regeneration in certain tissues by controlling the stem cell compartment, and it has also been shown to cooperate with the YAP/ TAZ pathway during colonic tissue repair and regeneration (36, 42). Furthermore, elevated NF- $\kappa B$ activation in combination with signals from the inflammatory microenvironment has been shown to increase WNT activation and lead to dedifferentiation of nonstem cells in tumor-initiating and cancer stem cell entities (43-45). This could explain the acquisition of the CD44v6 stem-like phenotype of the proliferative cyclin A2-deficient cells localized in the middle parts of the crypts. On the other hand, 1 study showed that cyclin A2 is involved in stabilizing the axin/GSK3 destruction complex, thereby promoting $\beta$-catenin degradation (46). This finding could explain the nuclear accumulation of $\beta$-catenin detected in 

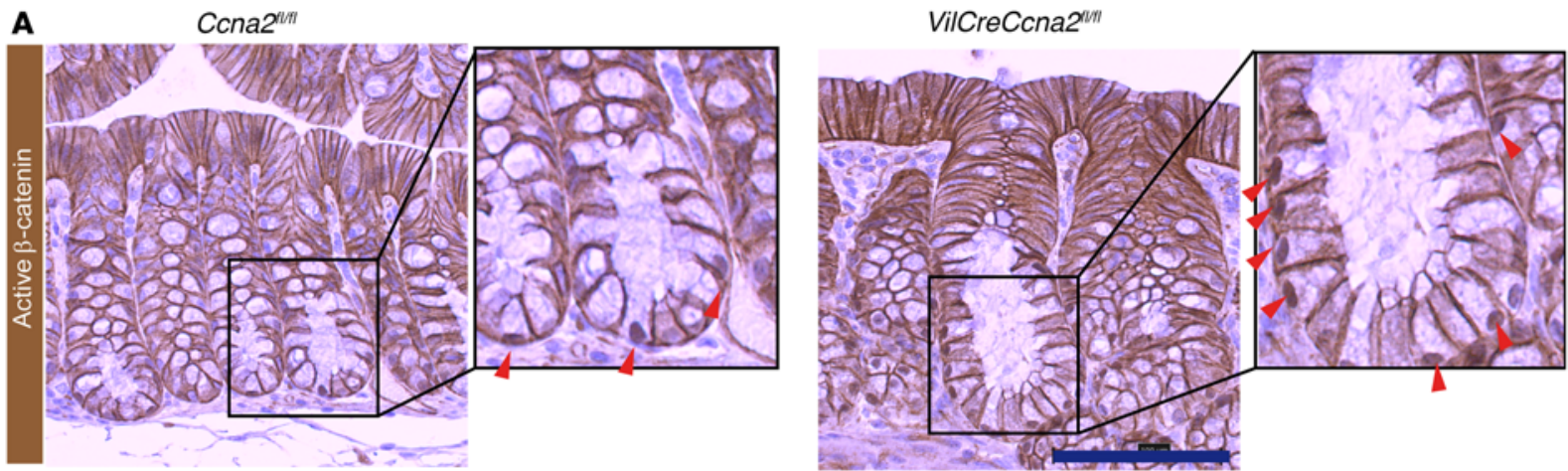

B

Day 2
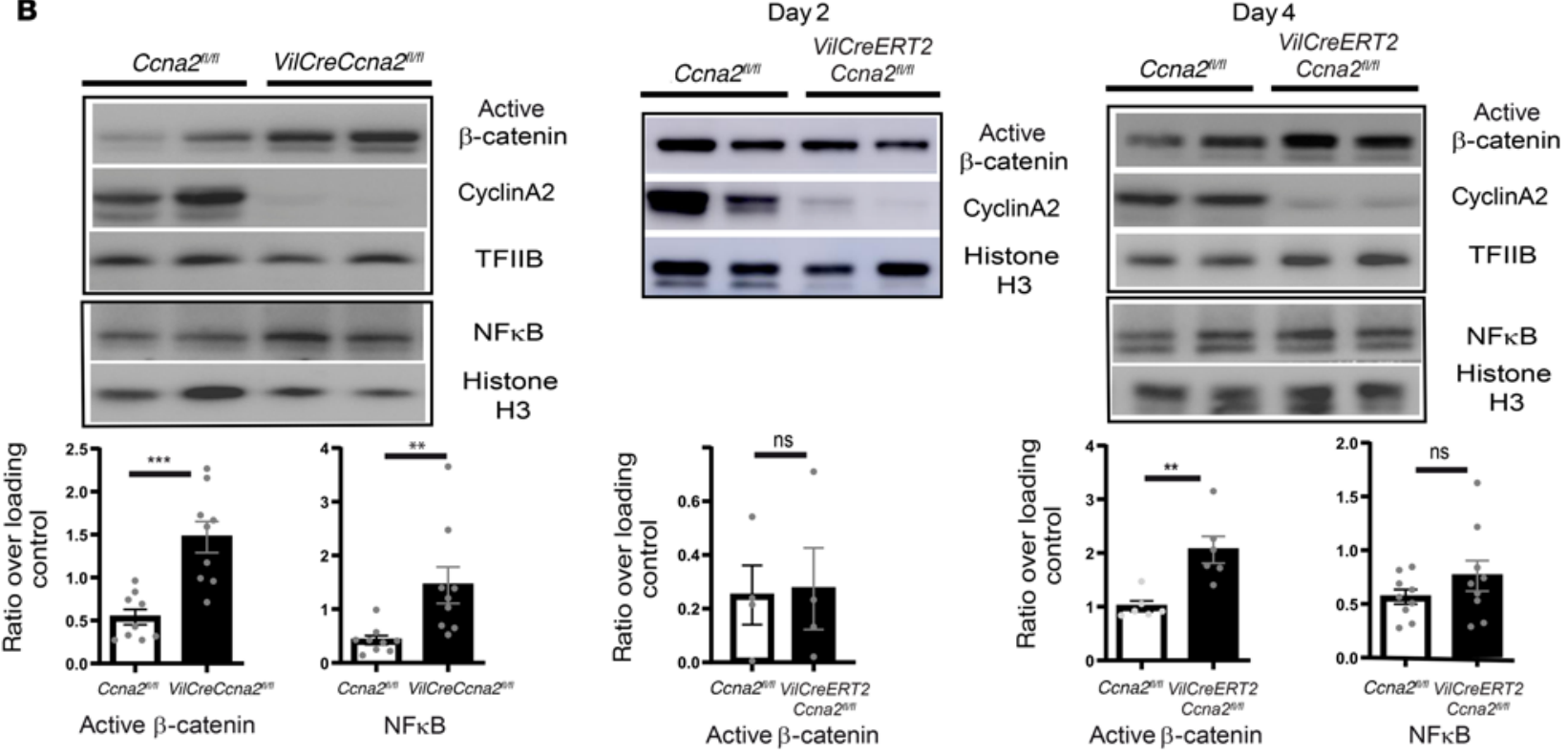

C
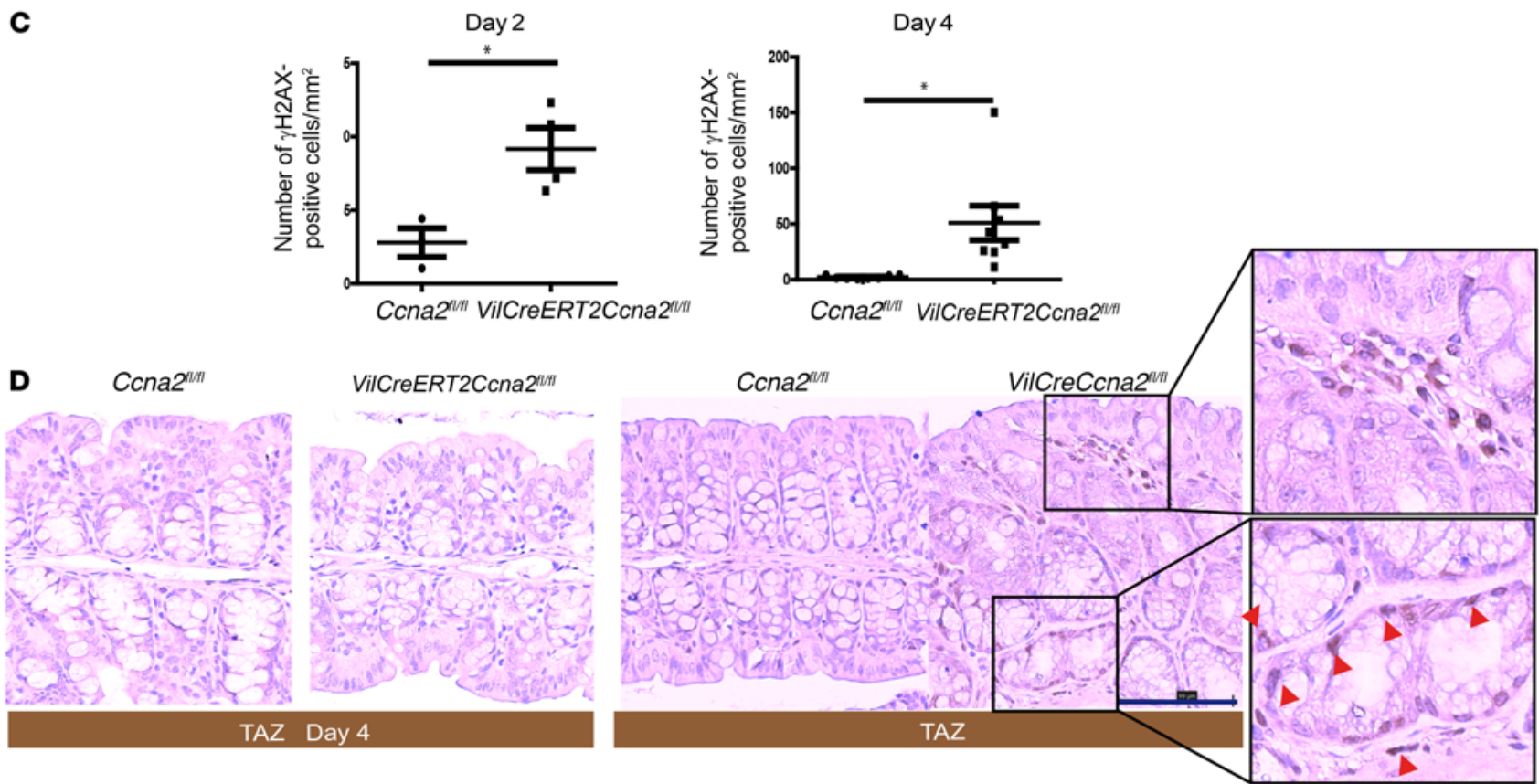
Figure 5. Cyclin A2 deficiency triggers regenerative pathways. (A) Representative immunostainings for active $\beta$-catenin in control (Ccna $\left.2^{f / / f}\right)$ and constitutive KO (VilCre Ccna2 ${ }^{f / f f}$ ) mice. Arrowheads indicate nuclear localization of active $\beta$-catenin. Scale bar: $100 \mu \mathrm{m}$. Original magnification, $\times 2$ (enlarged insets). (B) Western blot analysis of active $\beta$-catenin and NF- $\kappa B$ in nuclear extracts of CECs derived from constitutive cyclin A2-deficient mice $(n=9)$, their littermate controls (left panel), and tamoxifen-induced KO mice (VilCreERT2 Ccna2 ${ }^{f / f} /$ ) following deletion of cyclin A2 on day 2 ( $n$ $=4$, middle panel) and day 4 ( $n=6$ for $\beta$-catenin and $n=9$ for NF- $\kappa B$, right panel). Lower panels show quantification of active $\beta$-catenin and NF-kB after normalization with loading controls (TFIIB or histone H3). (C) Colons of cyclin A2-deficient mice displayed DNA damage. Plots show quantification of $\gamma \mathrm{H} 2 \mathrm{AX}$ staining in colons of control $(n=3)$ and VilCreERT2 Ccna ${ }^{f / / f l}(n=4)$ mice on days 2 and $4(n=8)$ following tamoxifen-induced Ccna2 depletion. (D) Representative immunostainings of TAZ expression (indicated by arrowheads) in constitutive cyclin A2-deficient colons and induced KO mice on day 4 following Ccna2 deletion (left panel). Scale bar: $100 \mu \mathrm{m}$. Original magnification, $\times 2$ (enlarged insets). Data represent the mean $\pm \mathrm{SEM} .{ }^{*} P<$ $0.05,{ }^{*} P<0.01$, and ${ }^{* * *} P<0.001$, by unpaired, 2-tailed Student's $t$ test.

cyclin A2-deficient colons, as well. In line with this is our previous description that cyclin A2 depletion in murine mammary epithelial cells promotes $\beta$-catenin activity $(18,19)$.

A previous study established a role of cyclin A2 in DNA DSB repair by generating mutant mice bearing cyclin $\mathrm{A} 2-\mathrm{KO}$ and hypomorphic alleles resulting in lower cyclin A2 expression (21). These mice fail to upregulate the meiotic recombination 11 (Mre11) nuclease that causes impaired resolution of stalled replication forks, reduced repair of DNA DSBs, aberrant chromosome segregation, and increased spontaneous and chemically induced tumor development (21). The same study reported that cyclin A2 binds to Mre11 mRNA, thereby promoting its translation. We detected increased Mre11 foci formation in cyclin A2-deficient CECs, suggesting that the increased DNA damage detected in the colons of cyclin A2-deficient mice resulted rather from a defective regulation of DNA replication and/or chromosome segregation. Indeed, phosphorylation of CDC6 by cyclin A/CDK2 has been shown to prevent re-replication during the $S$ phase and $G_{2}(47)$. Moreover, cyclin A2/CDK activity has been demonstrated to be essential for centrosome duplication and proper sister chromatid segregation (48). Of note, the mitoses detected in VilCre Ccna2 $2^{f / f l}$ mice were mostly located in the middle part of the colonic crypts, whereas $\gamma \mathrm{H}_{2} \mathrm{AX}^{+}$cells were predominantly identified in the upper part. This is in accordance with the fact that the generation of DSBs following abnormal mitosis takes place once a cell exits the mitotic process, entering the next cell cycle (49) thus during its migration toward the top part of the crypts. Notably, high expression levels of $\gamma \mathrm{H} 2 \mathrm{AX}$ have been shown to predict a poor prognosis for patients with CRC (50), and a more recent report described a role for cyclin A2 in preventing chromosomal defects (chromosomal instability [CIN]) in colon cancer cells (51).

It is well established that there is a crosstalk between DNA damage and inflammation and that either event can initiate the other one (52). Colons of cyclin A2-deficient mice displayed different signs of inflammation with infiltration of macrophages as well as production of IL- 6 and TGF- $\beta$, which correlated with increased activation of NF- $\kappa \mathrm{B}$ in CECs. Recent findings suggest a direct relationship between chromosome mis-segregation, DNA damage, and the induction of a proinflammatory environment (52). In fact,
DNA DSBs were shown to promote the production of IL- 6 and the TGF- $\beta$ family ligands $(53,54)$. Altogether, this suggests that the inflammatory response in cyclin A2-deficient mice is mounted as well in response to active genomic instability.

A large proportion of macrophages expressed YM1, indicating a predominant M2 polarization (Supplemental Figure 1C). Notably, M2-like macrophages have been described to promote tissue repair and remodeling and were attributed a protumoral role that could contribute to the phenotype of the cyclin A2-deficient mice (55). In addition, rectal prolapse was detectable in VilCre Ccna2 $2^{f / f l}$ mice, a phenomenon that was previously described in a mouse model that develops colitis spontaneously (28). Increased expression and activation of NF- $\mathrm{KB}$ has been observed in IBD and in patients with CRC, especially in mucosal macrophages and epithelial cells. This is accompanied by the production of cytokines including IL- 6 and TGF- $\beta$, which have been proposed as therapeutic targets for the treatment of $\operatorname{IBD}(26,56)$. Finally, YAP/TAZ overexpression has been shown to promote tumor formation (57) and to correlate with poor prognosis in CRC (58). We therefore conclude that DNA damage, combined with uncontrolled activation of several regenerative pathways, promotes the development of dysplasia in cyclin A2-deficient mice.

Most studies evaluating the prognostic value of cyclin A2 in CRC concluded that cyclin A2 overexpression is an indicator of poor prognosis (59-61), while 1 report described a higher survival rate for patients with tumors expressing high levels of cyclin A2 (62). To clarify the prognostic value of cyclin A2 in CRC, we performed a metaanalysis of 11 publicly available transcriptome data sets comprising 2239 primary tumor samples. This analysis revealed elevated CCNA2 transcript levels in primary tumors compared with levels in normal tissues as well as metastases, concurring with our previous analysis of biopsies using immunohistochemical and Western blot analysis (17). CCNA2 transcript expression was higher in tumors of patients with stage 1 or 2 CRC compared with expression levels in patients with stage 3 or 4 disease. Importantly, this concurred with cyclin A2 protein expression analysis by IHC on our TMA. Here, we show that CCNA2 expression differed among CMS subtypes, with the highest expression levels in the CMS1 subtype and the lowest in the CMS4 subtype. We stratified the transcriptome samples from high and nonhigh expressers by setting a cutoff of 2 or higher for the CCNA2 transcript expression ratio of $\mathrm{CRC}$ versus normal tissue and found higher RFS rates in the CCNA $2^{\text {hi }}$ class. Importantly, this favorable prognostic value of high CCNA2 expression persisted in multivariate analysis incorporating the classical prognostic factors or the CMS subtypes, suggesting an independent prognostic value. Whole-transcriptome supervised analysis of the two CCNA2 classes revealed that the 20 most significant modulated genes upregulated in the $C C N A 2^{\text {hi }}$ versus the CCNA $2^{\text {non-hi }}$ classes were all involved in cell proliferation and DNA repair. Although the expression data for multicellular tumor tissues cannot be directly correlated with the data obtained for cell typespecific $\mathrm{KO}$ mice, it is remarkable that $C C N A 2^{\text {hi }}$ CRC tumors and cyclin A2 deficiency in murine CECs triggered similar mechanisms such as DNA damage. We also compared the GES of the RNA-Seq

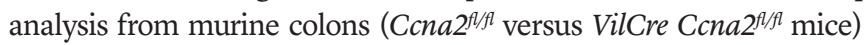
with the gene list used to establish the different CMS subtypes and found an overlap of 39 genes, among which 25 (>64\%) belonged to the CMS4 subtype (Supplemental Figure 13). Most of these genes 
A

B
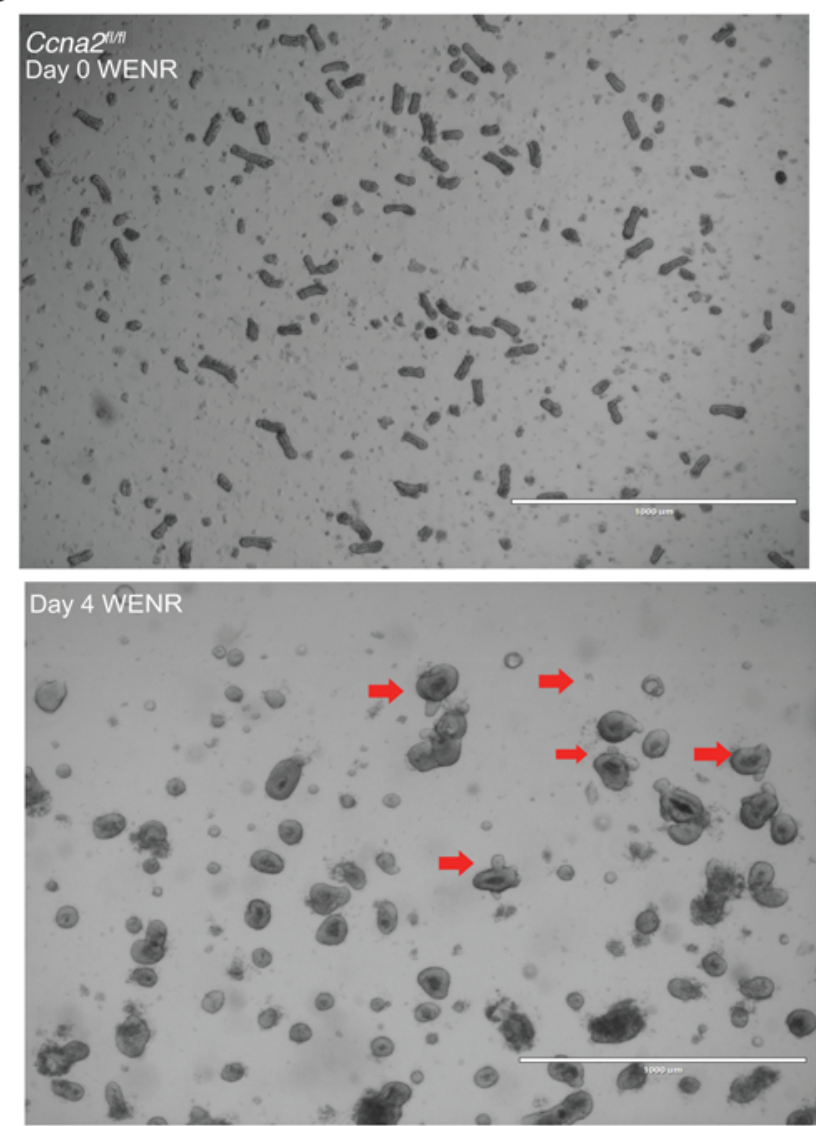

Figure 6. Cyclin A2-deficient organoids form cysts and only bud in the absence of WNT3a. (A)

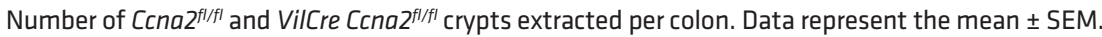
${ }^{*} P<0.01$, by unpaired, 2-tailed Student's $t$ test. (B) Representative images of crypts extracted

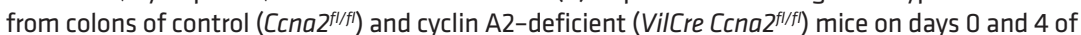
culturing in WNT3a-containing (WENR) media. Red circles indicate disrupted crypts. On day 4 of culturing, control organoids (left panel) were able to bud (indicated by red arrows), whereas cyclin A2-deficient organoids mainly formed cysts (right panel). Scale bars: $1 \mathrm{~nm}$. (C) Representative images of VilCre Ccna2 $2^{f / f / t}$ organoids on day 6 of culturing in WENR media (cyst formation) or in the absence of WNT3a (ENR) media (budding, indicated by red arrows). Scale bars: $1 \mathrm{~nm}$.

C

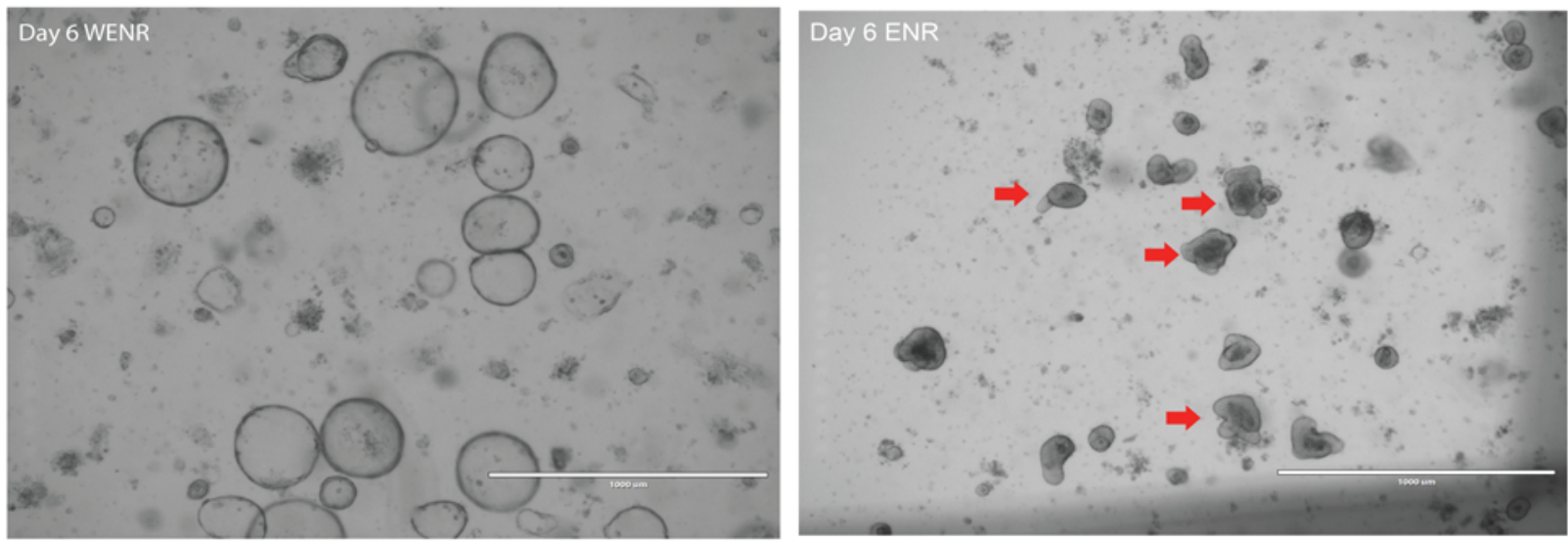


are known targets of TGF- $\beta$. This enrichment was significant (Fisher's exact test, $\left.P=5.10^{-8}\right)$. The CMS4 subtype, considered the "mesenchymal" class, is indeed defined by prominent TGF- $\beta$ activation, stromal invasion, and angiogenesis and is characterized by a dismal survival outcome. Notably, the invasive adenocarcinoma we detected upon cyclin A2 deletion in the CAC-treated mice displayed CMS4 features, such as stemness and TGF- $\beta$ expression. We conclude that the expression pattern of cyclin A2 in CRC tissues mirrors distinct roles during colon carcinogenesis, such as driving cell proliferation in early stages, when highly expressed, but promoting aggressiveness in later stages, when expression levels are lower.

Taken together, our results establish that cyclin A2 deficiency in murine CECs induced DNA damage, inflammation, and activation of different regenerative pathways triggering dysplasia. This rendered the mice more susceptible to chemically induced colon carcinogenesis by elevated tumor initiation as well as aggressiveness. Furthermore, cyclin A2 deletion in established tumors appeared to increase their aggressiveness. Our observations in mice were complemented by a meta-analysis of 2239 pCRC tumors, which showed that high CCNA2 expression is associated with a better prognosis in patients with CRC. Moreover, we describe here for the first time to our knowledge that CCNA2 expression differs between CMS subtypes. Importantly, classification of the patient cohort into the different CMSs identified CCNA2 $2^{\text {non-hi }}$ expression as a poor prognostic factor within CMS1, CMS3, and CMS4, but not CMS2. In conclusion, CCNA2 expression was strongly correlated with several clinicopathological factors of CRC and may represent a favorable prognostic marker in patients with CRC.

\section{Methods}

Generation of cyclin A2 conditional-KO mice in the intestinal epithelium. To generate cyclin A2 intestinal epithelium-specific KO mice, Ccna2 $2^{f / f l}$ mice, provided by P. Sicinski (Dana Farber Institute, Boston, Massachusetts, USA) (23), were crossed with transgenic mice carrying either a constitutively active Cre recombinase (vil-Cre) (provided by Sylvie Robine, Curie Institute, Paris, France) (24), expressed during embryonic development (day 9) throughout adulthood in intestinal epithelial cells, or a tamoxifen-inducible Cre under the same promoter (vil-Cre-ER ${ }^{T 2}$ ) (provided by Robine, Curie Institute, Paris, France) (24), induced in adult mice by 2 intraperitoneal injections of tamoxifen (MilliporeSigma, $150 \mathrm{mg} / \mathrm{kg}$ ). Mice were maintained on a C57BL6 background. Genotyping was performed using the primers listed in Supplemental Table 9.

Immunohistochemical and immunofluorescence analyses of paraffin-embedded tissue sections. Colons were rolled up lengthwise and embedded in paraffin after fixation in $10 \%$ formalin for 24 hours. Tissue slides were deparaffinized and rehydrated in xylene $(2 \times 5 \mathrm{~min})$, $100 \%$ ethanol ( $5 \mathrm{~min}), 96 \%$ ethanol ( $3 \mathrm{~min}), 70 \%$ ethanol $(3 \mathrm{~min})$, and $\mathrm{dH}_{2} \mathrm{O}$ (5 min). Tissue slides were incubated in $10 \mathrm{mM}$ sodium citrate, pH6.0, for 20 minutes at $100^{\circ} \mathrm{C}$ or in $10 \mathrm{mM}$ Tris, $1 \mathrm{mM}$ EDTA, pH 9.0, for antigen retrieval, depending on the antibody. Following 2 washes in PBS-0.2\% Tween 20, tissue slides were incubated in PBS with 3\% hydrogen (H1009, MilliporeSigma) for 5 minutes at room temperature, blocked in $2.5 \%$ blocking serum, $5 \%$ BSA, and 5\% nonfat milk for 30 minutes at room temperature, and then incubated with a primary antibody for 1 hour at $4^{\circ} \mathrm{C}$. The corresponding secondary antibody reagents (ImmPRESS Kit, Vector Laboratories) were used for detection. The primary antibodies used are summarized in Supplemental Table 10.
For H\&E staining, tissue slides were incubated in xylene $(3 \times 3$ $\mathrm{min}$ ), $100 \%$ ethanol $(3 \times 3 \mathrm{~min})$, $95 \%$ ethanol $(3 \mathrm{~min}$ ), and $80 \%$ ethanol $(3 \mathrm{~min})$ and then for 5 minutes in deionized water. Slides were then incubated in hematoxylin (Vector Labratories) for 3 minutes, extensively rinsed in tap water, and dipped in acid ethanol $(0.03 \mathrm{~N} \mathrm{HCl}$, $70 \%$ ethanol). After rinsing in water, the slides were transferred into deionized water for 2 minutes and then stained in eosin for 30 seconds (Vector Laboratories), followed by 3 incubations of 5 minutes each in 95\% ethanol. After incubations in xylene, the coverslips were mounted using Permount Mounting Medium (Thermo Fisher Scientific). For TMA analysis, the rabbit anti-cyclin A2 antibody (ab181591, Abcam), validated on Ccna2-deficient mouse tissues, as well as the rabbit anticyclin A2 antibody (NCL-CYCLINA) were used (Supplemental Table 10). Slides were scanned using the Nanozoomer scanner (Hamamatsu Photonics) and analyzed in NDP Viewer with gamma settings modified to accommodate for interslide variability of the HE staining.

For immunofluorescence analysis, the tissue slides were deparaffinized and rehydrated as described above and incubated in $1 \mathrm{mM}$ EDTA antigen retrieval buffer (pH 9.0) for $\alpha$ - and $\gamma$-tubulin, or in $10 \mathrm{mM}$ citrate ( $\mathrm{pH}$ 6) for anti-Mre11 staining. Blocking was performed for 30 minutes in $2.5 \%$ blocking serum, $5 \%$ BSA, and $5 \%$ nonfat milk at room temperature, and tissue slides were then incubated with primary antibodies (see Supplemental Table 10) for 1 hour at $4^{\circ} \mathrm{C}$. Depending on the primary antibodies, slides were incubated with goat anti-mouse, -rat, or -rabbit secondary antibodies conjugated to either Alexa Fluor 488 or Alexa Fluor 555 (Thermo Fisher Scientific) in addition to DAPI (0.1 $\mu \mathrm{g} / \mathrm{mL}$ ) for 1 hour at room temperature. Mre11 staining images were acquired on the Leica Confocal SP5-SMD, and images were analyzed using Imaris software (Oxford Instruments). For costaining (i.e., $\alpha$ - $/ \gamma$-tubulin, Ki67/active $\beta$-catenin, Ki67/H2AX, and Ki67/CD44v6), image acquisition in the transverse and proximal colon was performed using Metamorph software (Molecular Devices) on the Upright Zeiss Axioimager Z2 (Zeiss), and images were analyzed using ImageJ software.

BrdU incorporation assay. Mice were intraperitoneally injected with $100 \mu \mathrm{g} / \mathrm{g}$ BrdU (B9285, MilliporeSigma) diluted in PBS. Colon specimens were dissected 2 hours after injection, flushed with cold PBS, and embedded in paraffin after fixation in 10\% formalin for 24 hours. Incorporation of BrdU was detected using an anti-BrdU antibody (Supplemental Table 10) after deparaffinization of the tissues, antigen retrieval in citrate buffer, and DNA denaturation using $2 \mathrm{~N} \mathrm{HCl}$ for 1 hour at $37^{\circ} \mathrm{C}$ followed by an incubation in $0.1 \mathrm{M}$ borax buffer, pH9. Revelation was performed using the VECTASTAIN Avidin-Biotin Kit (Vector Laboratories).

Isolation of CECs. Colons were removed from mice, flushed with cold PBS, cut longitudinally, and treated with $10 \mathrm{~mL}$ CEC buffer (PBS, 1\% BSA, $1 \mathrm{mM}$ EDTA, $1 \mathrm{mM}$ DTT, $5.6 \mathrm{mM}$ glucose) at $37^{\circ} \mathrm{C}$ for 45 minutes under continuous shaking to release CECs. Cell purity was validated by FACS using the cell-surface markers EpCAM-APC and CD45-PE and CD90.2-FITC (see Supplemental Table 10) to identify epithelial and immune cells, respectively.

Isolation of CEC cytoplasmic and nuclear fractions. Cells were treated with ice-cold hypotonic buffer (10 mM HEPES, pH 7.9, $10 \mathrm{mM} \mathrm{KCl,}$ O.1 mM EDTA, 0.1 mM EGTA, 0.5 mM PMSF, 1 mM DTT) and cOmplete Inhibitor Tabs (MilliporeSigma) for 15 minutes, and then treated with $10 \%$ NP40 and vortexed. The nuclei were pelleted by centrifugation (16.1 rcf, $15 \mathrm{~s}$ ) while the supernatant was kept as the cytoplasmic fraction. Nuclei were lysed in $20 \mathrm{mM}$ HEPES, pH 7.9, $0.4 \mathrm{mM} \mathrm{NaCl}$, 1 mM EDTA, 1 mM EGTA, 0.5 mM PMSF, 1 mM DTT, and cOmplete 
A
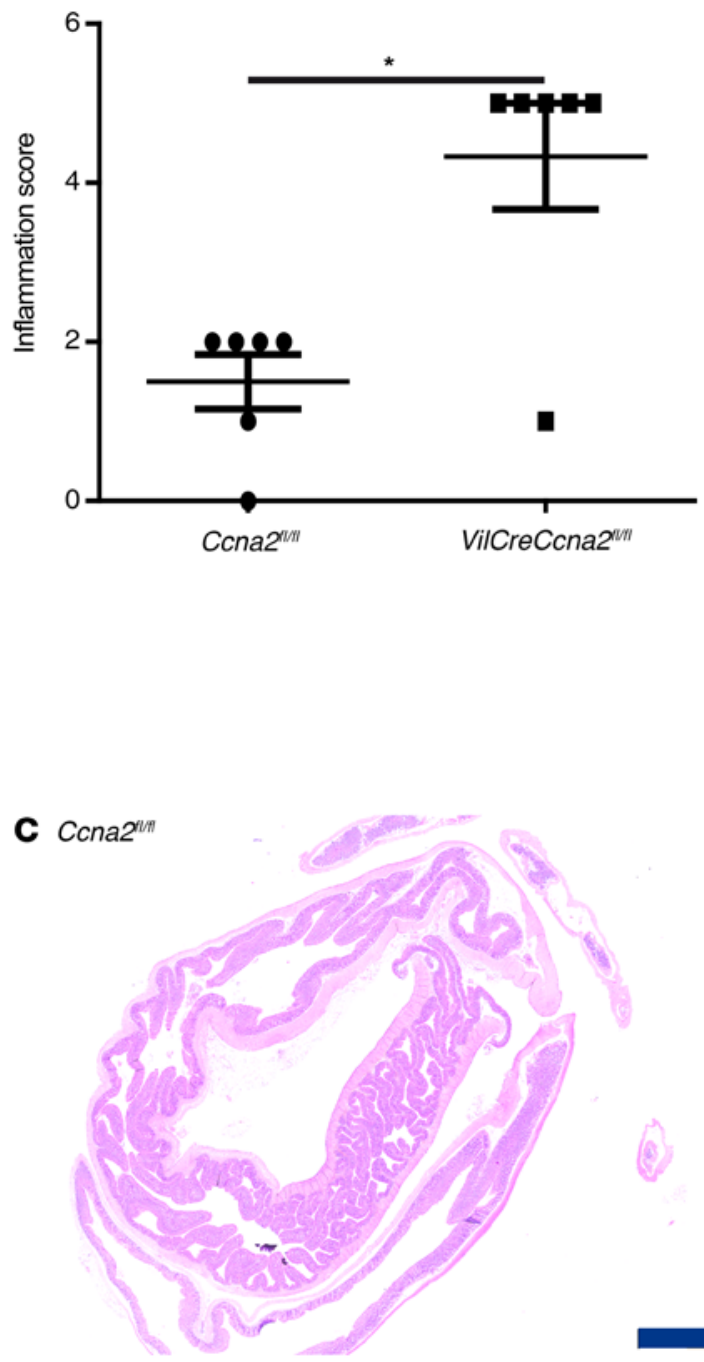

B
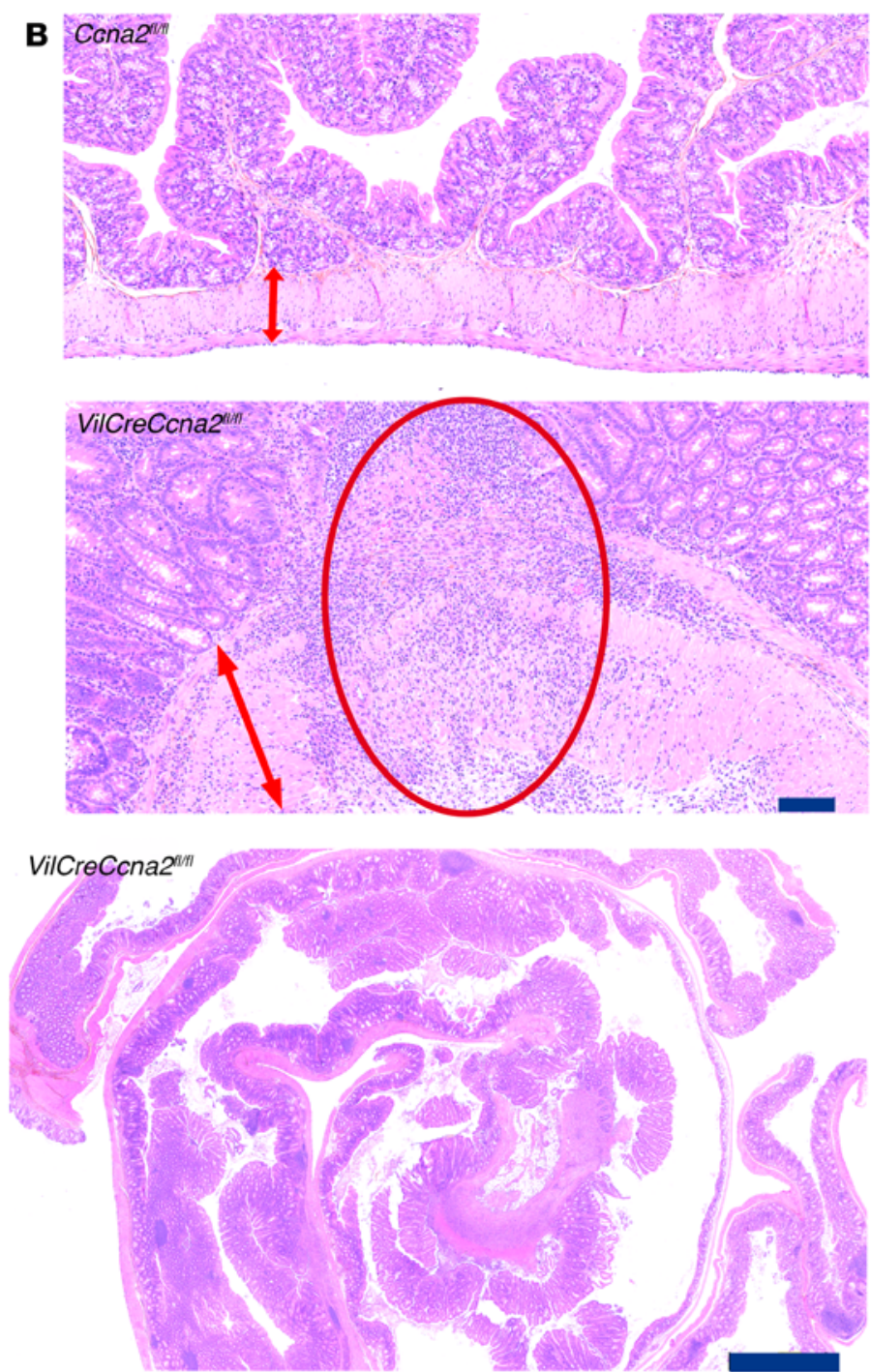

D

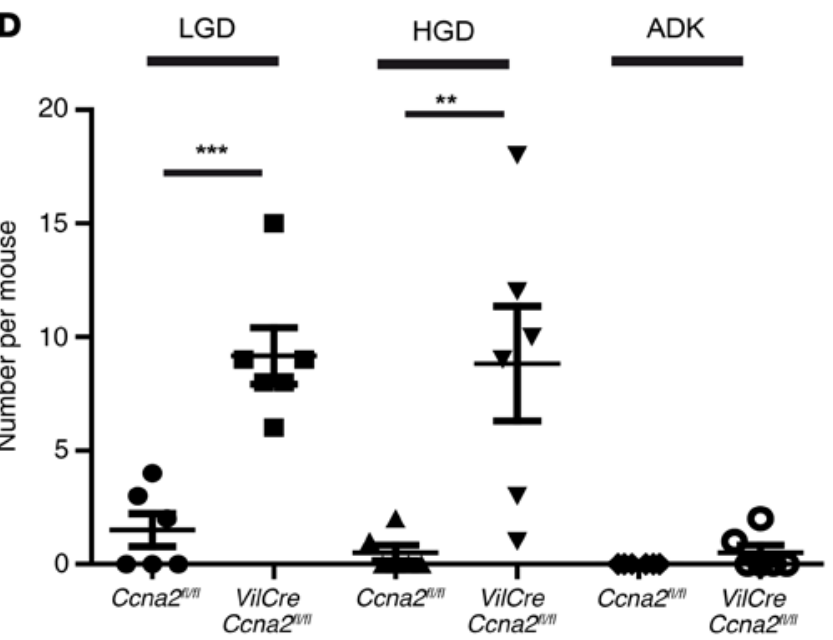

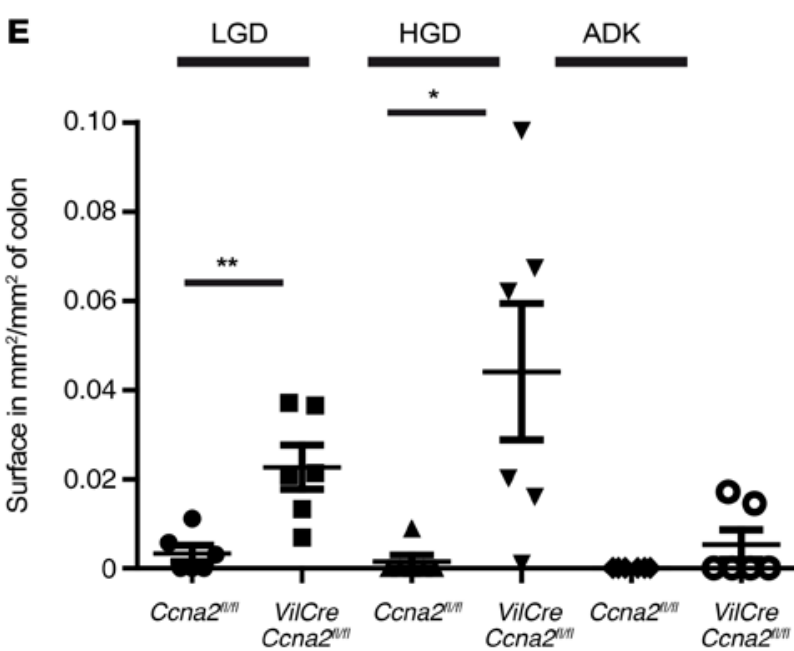

Figure 7. Cyclin A2-deficient mice are more susceptible to CAC. CCna $2^{f / f l}(n=6)$ and VilCre Ccna2 $2^{f / f l}(n=7)$ mice were subjected to a modified AOM/DSS protocol including only 1 DSS treatment (Supplemental Figure 6A). (A and B). Quantification of the inflammatory scores for colons from control and VilCre CCna $2^{f / f l}$ mice (A) and representative $\mathrm{H} \& \mathrm{E}$ stainings showing transmural immune cell infiltration (indicated by the red oval) in KO mice (B) (the red arrows mark the muscularis mucosa). Scale bar: $100 \mu \mathrm{m}$. (C) Representative images of H\&E-stained colons from control and cyclin A2-deficient mice subjected to the CAC protocol. Scale bars: $2 \mathrm{~mm}$. (D and E) Number of dysplasia (LGD, HCD]) and adenocarcinoma (ADK) occurrences per mouse and dysplasia and adenocarcinoma area expressed in $\mathrm{mm}^{2} / \mathrm{mm}^{2}$ of colon in control and VilCre Ccna2 ${ }^{f / f l}$ mice. Data represent the mean $\pm \mathrm{SEM}$. ${ }^{*} P<0.05$, ${ }^{* *} P<0.01$, and ${ }^{* * *} P<0.001$, by unpaired, 2-tailed Student's $t$ test. 

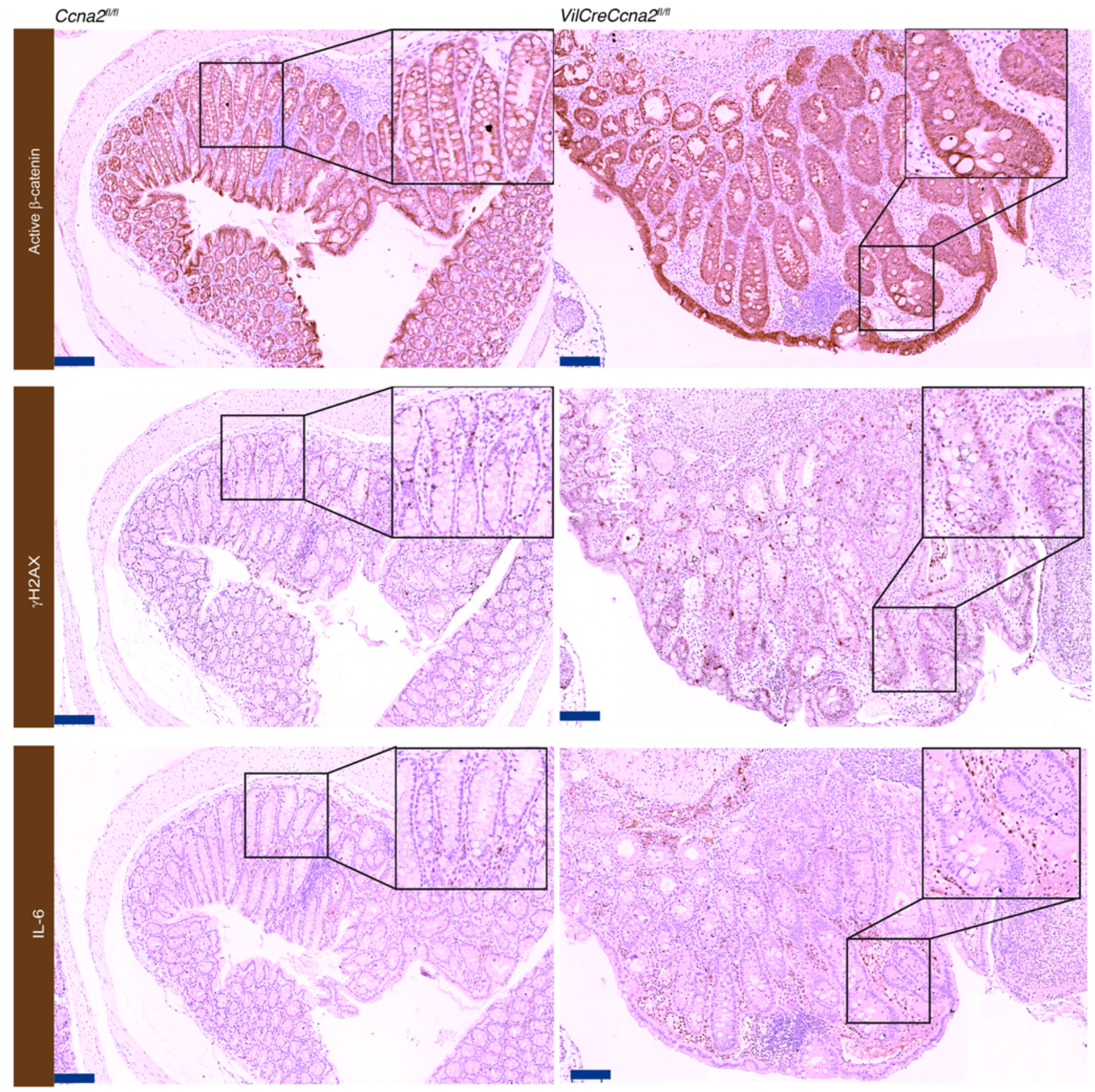

Figure 8. Lesions in colons from cyclin A2-deficient mice exposed to CAC display elevated levels of active $\beta$-catenin, DNA damage, and IL-6 expression. Representative immunostainings for active $\beta$-catenin, $\gamma \mathrm{H} 2 \mathrm{AX}$, and IL-6 in morphologically similar lesions in colons from control and cyclin A2-deficient mice at the end of the AOM/DSS protocol described in Supplemental Figure 10A. Scale bars: $100 \mu \mathrm{m}$. Original magnification, $\times 1.5$ (enlarged insets).

Inhibitor Tabs (MilliporeSigma). The supernatant was collected as the nucleoplasmic fraction after centrifugation (16.1 rcf, $5 \mathrm{~min}$ ).

Western blot analysis. Protein samples were prepared in $4 \times$ Laemmli buffer, separated in sodium dodecyl sulfate-polyacrylamide gels, and electro blotted onto nitrocellulose membranes (MilliporeSigma) using a wet/tank transfer system (Bio-Rad). The antibodies used for Western blotting in this study are listed in Supplemental Table 10. All the experiments were performed at least 3 times in triplicate. Quantification of immunoblots was carried out using ImageJ. Experimental values are expressed as the ratio of protein levels versus control with the TFIIB and histone H3 signal for the nucleoplasmic fraction. The complete unedited blots are shown in the supplemental material.

Colon organoids. Colons were washed extensively in cold PBS and penicillin-streptomycin and then cut into $0.5 \mathrm{~cm}$ pieces, which were placed into a $50 \mathrm{~mL}$ tube containing $10 \mathrm{~mL}$ cold PBS. The pieces were washed 5 to 10 times with cold PBS supplemented with antibiotics and then incubated in PBS and $25 \mathrm{mM}$ EDTA on a roller for 30 minutes at $4^{\circ} \mathrm{C}$. After sedimentation, the pieces were successively washed 4 times 
A
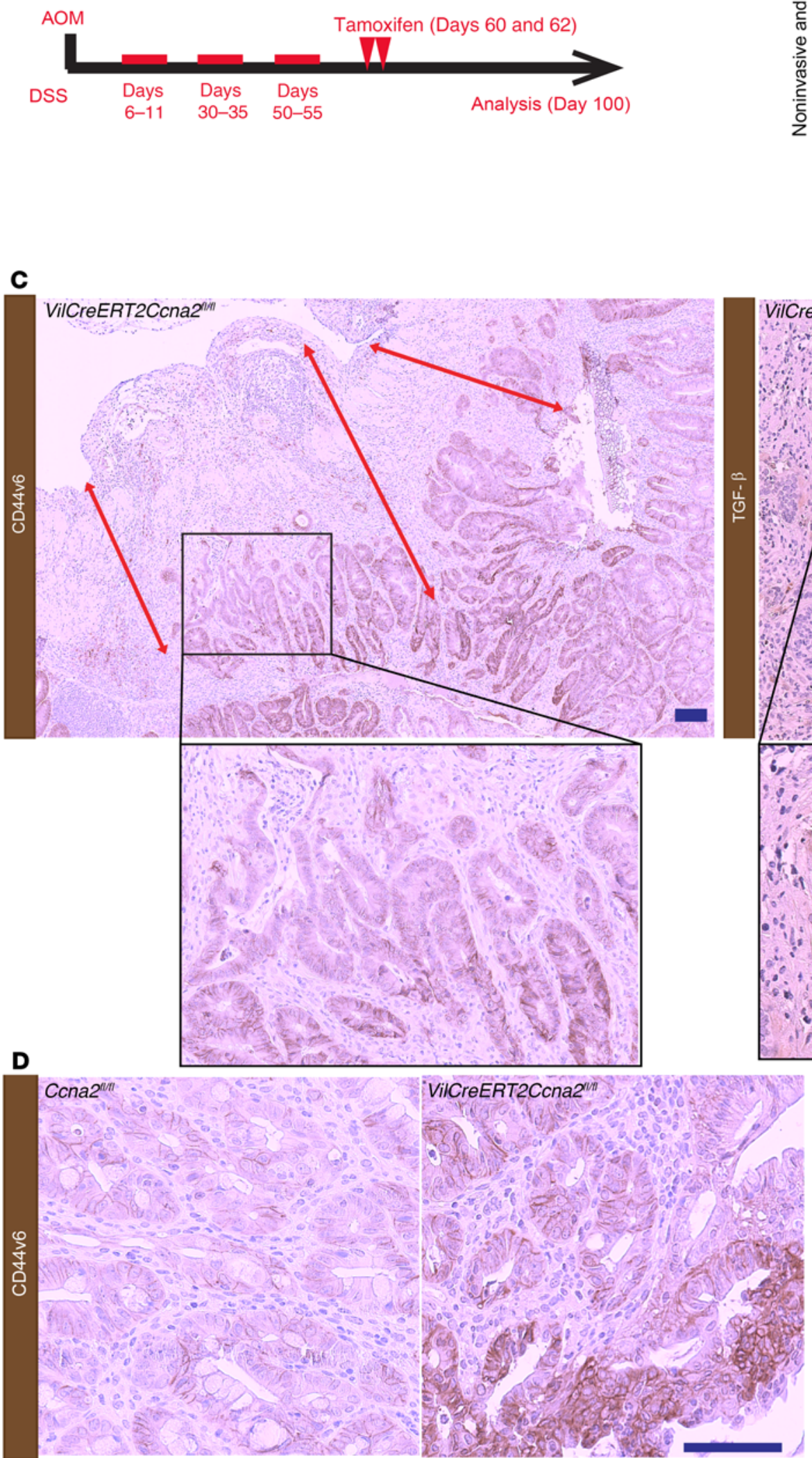
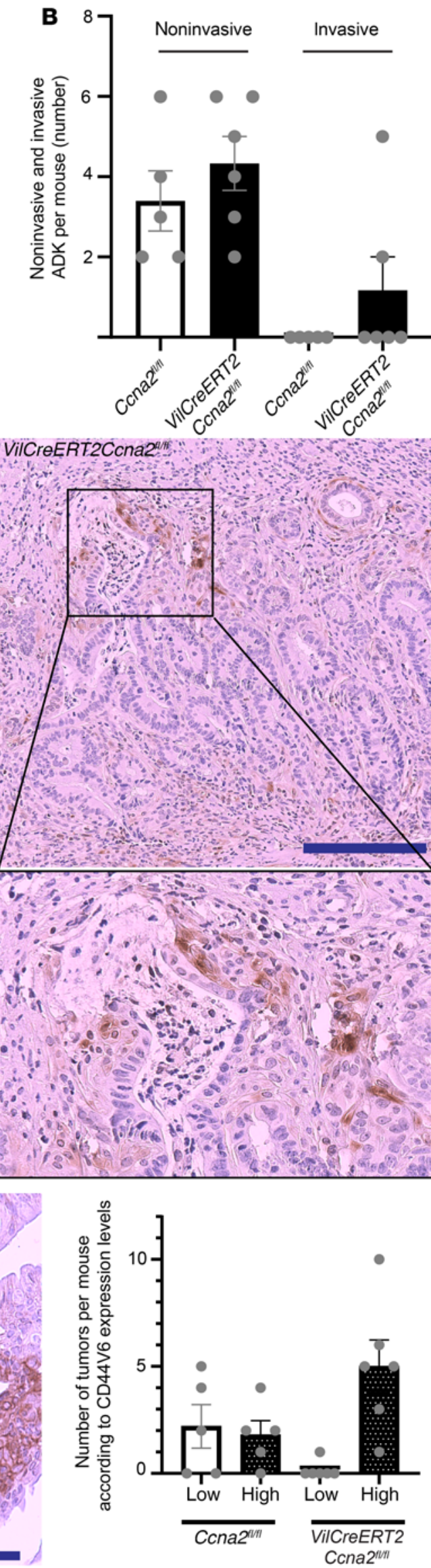
Figure 9. Inactivation of cyclin A2 on established tumor lesions leads to invasive adenocarcinomas. (A) Schematic representation of the AOM/ DSS protocol applied to VilCreERT2 Ccna2 $2^{f / f l}(n=8)$ and control $(n=8)$ mice. Note that cyclin A2 deletion was induced by tamoxifen injection after the last DSS treatment. (B) Quantification of the number of noninvasive versus invasive adenocarcinomas per mouse for control (Ccna2 $\left.2^{f / f}\right)$ and cyclin A2deficient (VilCreERT2 (cna2 fl/f) mice ( $n=8$ each). (C) Representative images of an invasive adenocarcinoma disrupting the muscularis mucosa and infiltrating through the muscularis propria, as indicated by red lines. The panel on the left shows an immunostain for CD44v6 in tumor cells, and the panel on the right shows a focal immunostain for TCF- $\beta$ in the desmoplastic stroma surrounding the tumor cells. Scale bars: $100 \mu \mathrm{m}$. Original magnification, $\times 3$ (enlarged insets). (D) Representative images of immunostains for CD44v6 on tumors of control and cyclin A2-deficient mice and corresponding quantification of low and high CD44v6-expressing tumors. Tumors were classified as low expressers when less than $50 \%$ of the area had detectable CD44v6 expression. Scale bar: $100 \mu \mathrm{m}$. Data represent the mean \pm SEM.

in $10 \mathrm{~mL}$ cold PBS. The supernatant for each wash was strained in a 70 $\mu \mathrm{m}$ mesh filter, and each fraction (F1-F4) was centrifuged at $600 \mathrm{~g}$ for 5 minutes and checked under the microscope for the presence of wellformed crypts. In most cases, fractions 3 and 4 were used for the organoid culture. A total of 200 crypts were resuspended in $50 \mu \mathrm{L}$ Matrigel

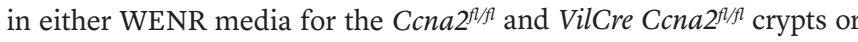
ENR media for the VilCre Ccna $2^{f / f l}$ crypts (Supplemental Table 11) and microscopically inspected once a day.

RNA-Seq sample preparation and bioinformatics analysis. CECs were isolated from the proximal colons of 4 -week-old Ccna $2^{f / f l} \mathrm{Vil}$ Cre (KO) and Ccna2 $2^{f / f l}$ (control) mice, as described above. RNA was extracted using the Roche High Pure RNA Isolation Kit (Roche Molecular Systems), and purity as well as integrity were controlled using the Agilent RNA 6000 Nano Kit (Agilent Technologies). RNA-Seq was performed on an Illumina NextSeq 500 platform. Quantification of the gene transcripts was performed using the Kallisto program (63), which is based on the pseudoalignment principle, with default settings. GRCm38 RefSeq was used to annotate the transcripts. We used the STAR program (64) to align the reads on the Mus musculus reference genome (GRCm38/mm10). Raw data are available in the NCBI's Gene Expression Omnibus (GEO) database (GEO GSE149716; https://www. ncbi.nlm.nih.gov/geo/query/acc.cgi?acc $=$ GSE149716). For differential expression analysis (DEA), we first filtered the transcripts with too-low counts among the samples using the filterByExpr function of edgeR, with default settings. To perform the data normalization and DEA of Ccna2-deficient versus control samples, we ran standard steps of edgeR and DESeq2 in parallel on the filtered data. For both DEA methods, we applied a cutoff threshold of 0.05 to the adjusted $P$ values and a cutoff threshold of 2 for the fold changes. The 2 resulting lists of significantly differentially expressed genes were intersected. We separated the upregulated and downregulated genes on this unique list. Finally, to perform functional enrichment analysis, these sublists were submitted to the dedicated function of the clusterProfiler package, available on Bioconductor (https://bioconductor.org/packages/ release/bioc/html/clusterProfiler.html). A Fisher's exact or $\chi^{2}$ test was used when appropriate to analyze the enrichment of the CCNA2 signature with the published signatures of interest.

CAC. For induction of CAC in the VilCre Ccna $2^{f / / l}$ mice and their corresponding controls, mice were intraperitoneally injected with 6.25 $\mathrm{mg} / \mathrm{kg}$ AOM (MilliporeSigma). Mice were treated with one 5-day cycle of $1.75 \%(\mathrm{w} / \mathrm{v})$ DSS (TdB Consultancy AB) in the drinking water on day 5 of the protocol. Mouse weights were checked every 2 days for 2 months, except for the week following the DSS treatment, when the weight was measured every day. Mice were then sacrificed and colons prepared for histological analysis after fixation in $10 \%$ formalin. Tissues were embedded in paraffin, and sections were stained with $\mathrm{H} \& \mathrm{E}$ or incubated with primary antibodies (anti-active $\beta$-catenin, anti- $\gamma \mathrm{H} 2 \mathrm{AX}$, and anti-IL-6), as reported above. Colitis was scored as outlined in Supplemental Table 3. For induction of CAC in the VilCreERT2 Ccna $2^{f / f l}$ mice and their controls, mice were injected as described above with AOM but were treated 3 times with $1.75 \%$ DSS in the drinking water for 5 days on days 6,30 , and 50 of the protocol. Tamoxifen intraperitoneal injection was performed on days 63 and 65 to induce cyclin A2 inactivation, and mice were sacrificed on day 100 for analysis.

Patient samples. Details of the TMA generated at Centre de Ressources de l'Institut Régional du Cancer de Montpellier (CRB-ICM) (agreement number BB-033-00059) in Montpellier, France, are listed in Supplemental Table 6. Some tissue samples were generated as duplicates from the same patients but were considered individual templates for the statistical analysis because of tumor heterogeneity.

For the transcriptome meta-analysis, we collected clinicopathological and gene expression data on tissues from NC, pCRC, and metastatic CRC (mCRC) samples from 10 public data sets and 1 data set generated at the Institut Paoli Calmettes (Marseille, France) comprising at least 1 probe set representing CCNA2. Sets and raw data were collected from the NCBI's GenBank and GEO, ArrayExpress, and TCGA databases (Supplemental Table 4). Samples were profiled using whole-genome DNA microarrays (Affymetrix) or RNA-Seq (Illumina). The analyzed data set contained a total of 2401 samples, including 95 NC samples, 2239 pCRC samples, and the $67 \mathrm{mCRC}$ samples included in the present analysis, which were obtained from liver $(n=47)$ and lung $(n=20)$.

Gene expression data analysis. Data analysis required a step of preanalytic processing. We first normalized each data set separately using the robust multi-array average (RMA) (65) with the nonparametric quantile algorithm for the raw Affymetrix data. Normalization was done in $\mathrm{R}$ using Bioconductor and associated packages. We then mapped hybridization probes across the different microarrays represented as previously reported (66). When multiple probes mapped to the same GeneID, the one with the highest variance in each data set was retained. The available TCGA RNA-Seq data that were already normalized were $\log _{2}$ transformed. Next, CCNA2 mRNA expression data were extracted and the batch effects corrected through the 11 studies using $z$ score normalization. Briefly, for each expression value in each separate study, CCNA2 values were transformed by subtracting the mean of the gene in that data set divided by its SD, measuring the mean and SD of primary samples. CCNA2 expression was measured as a discrete value after comparison with median expression in the $95 \mathrm{NC}$ samples; upregulation, thereafter designated CCNA2 $2^{\text {hi }}$, was defined by a CRC/NC ratio of 2 or higher, and no upregulation (CCNA2 ${ }^{\text {non-hi }}$ ) by a CRC/NC ratio below 2 . The CMS classification (8) was based on the CMScaller tool made by Eide et al. (67). Finally, to explore the biological pathways associated with our CCNA2-based classification, a supervised analysis was applied using learning and validation sets. The learning set included the 459 samples of TCGA data set that included 222 CCNA2 $2^{\text {non-hi }}$ and 237 CCNA2 $2^{\text {hi }}$ samples. A moderated Student's $t$ test with empirical Bayes statistic included in the limma R packages was used. The FDR (68) was applied to correct 

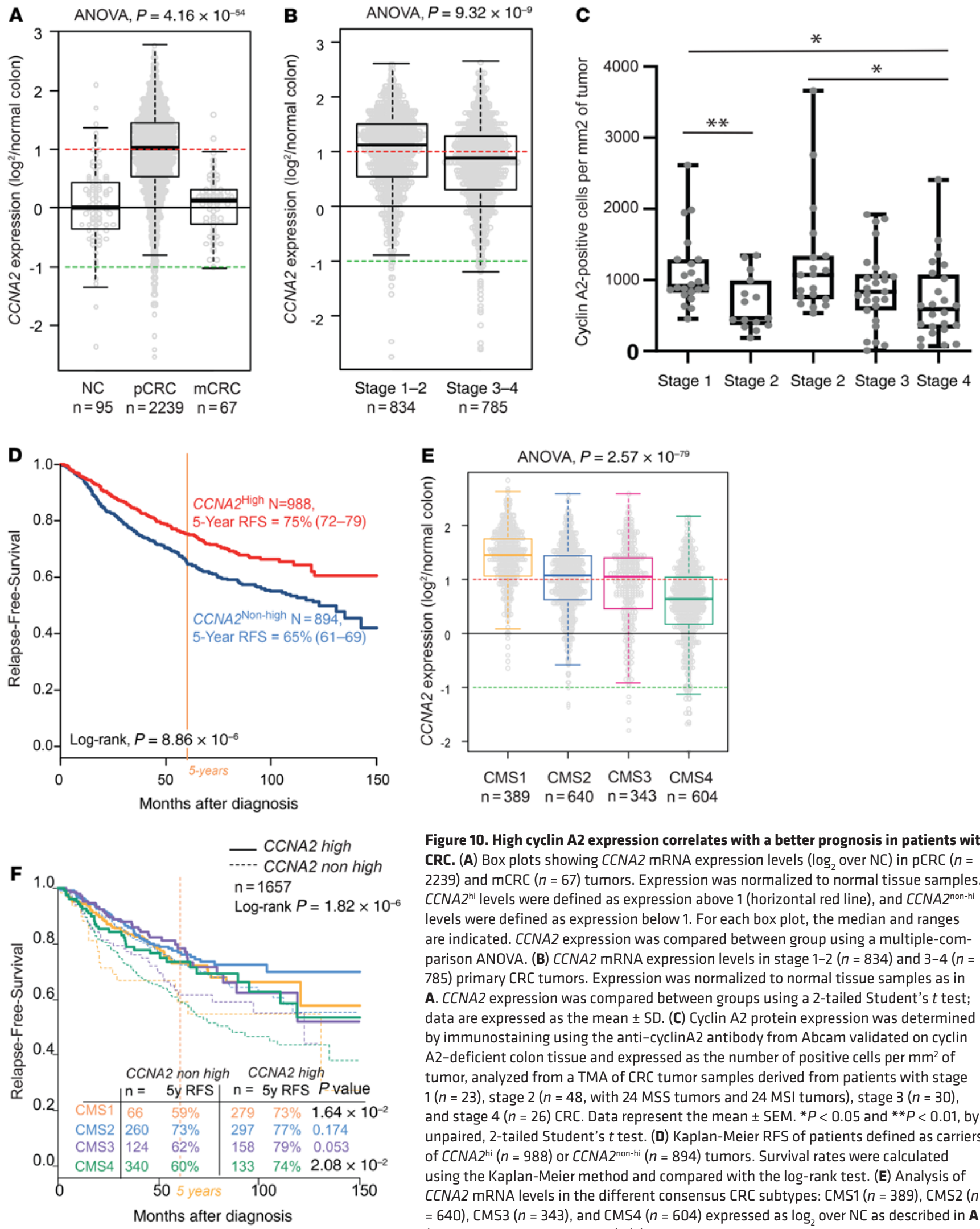

Figure 10. High cyclin A2 expression correlates with a better prognosis in patients with CRC. (A) Box plots showing CCNA2 mRNA expression levels ( $\log _{2}$ over NC) in pCRC ( $n=$ 2239) and mCRC ( $n=67)$ tumors. Expression was normalized to normal tissue samples. CCNA $2^{\text {hi }}$ levels were defined as expression above 1 (horizontal red line), and CCNA2 $2^{\text {non-hi }}$ levels were defined as expression below 1 . For each box plot, the median and ranges are indicated. CCNA2 expression was compared between group using a multiple-comparison ANOVA. (B) CCNA2 mRNA expression levels in stage 1-2 $(n=834)$ and 3-4 $(n=$ 785) primary CRC tumors. Expression was normalized to normal tissue samples as in A. CCNA2 expression was compared between groups using a 2-tailed Student's $t$ test; data are expressed as the mean \pm SD. (C) Cyclin A2 protein expression was determined by immunostaining using the anti-cyclinA 2 antibody from Abcam validated on cyclin A2-deficient colon tissue and expressed as the number of positive cells per $\mathrm{mm}^{2}$ of tumor, analyzed from a TMA of CRC tumor samples derived from patients with stage $1(n=23)$, stage $2(n=48$, with 24 MSS tumors and 24 MSI tumors), stage $3(n=30)$, and stage $4(n=26)$ CRC. Data represent the mean \pm SEM. ${ }^{*} P<0.05$ and ${ }^{* *} P<0.01$, by unpaired, 2-tailed Student's $t$ test. (D) Kaplan-Meier RFS of patients defined as carriers of CCNA2 $2^{\text {hi }}(n=988)$ or CCNA2 $2^{\text {non-hi }}(n=894)$ tumors. Survival rates were calculated using the Kaplan-Meier method and compared with the log-rank test. (E) Analysis of CCNA2 mRNA levels in the different consensus CRC subtypes: CMS1 $(n=389)$, CMS2 ( $n$ $=640)$, CMS3 $(n=343)$, and CMS4 $(n=604)$ expressed as $\log _{2}$ over NC as described in $\mathbf{A}$ (multiple-comparison ANOVA). (F) Kaplan-Meier RFS curves for all patients with pCRC according to both the CCNA2-based classification (CCNA2 ${ }^{\text {hi }}$ and CCNA2 ${ }^{\text {non-hi }}$ classes with the solid curves and dashed curves, respectively) and CMS subtypes. 
the multiple testing hypothesis, and significant genes were defined by the following thresholds: a $P$ value of less than $5 \%$, a $q$ value of less than $25 \%$, and a fold change of greater than 2 . The resulting GES was based on a metagene score defined as the difference between the mean expression of genes upregulated and the mean expression of genes downregulated in the CCNA $2^{\text {hi }}$ samples, using a cutoff of 0 . This score was then applied to both learning and validation sets to test the robustness of the GES (Student's $t$ test). Gene ontology (GO) analysis of the gene list was based on GO biological processes of the Database for Annotation, Visualization, and Integrated Discovery (DAVID) (http://david.abcc.ncifcrf.gov/).

Statistics. Correlations between the CCNA2 expression-based classes (non-high versus high) and the clinicopathological factors were calculated using the Fisher's exact test for binary variables and the Student's $t$ test for continuous variables. Our primary endpoint, RFS, was calculated from the date of diagnosis until the date of metastatic relapse or death from CRC. The follow-up was measured from the date of diagnosis to the date of last news for event-free patients. Survival was calculated using the Kaplan-Meier method, and curves were compared with the log-rank test. Univariate and multivariate analyses were done using Cox regression analysis (Wald's test). The variables tested in univariate analysis included the CCNA2-based classification (non-high versus high), patients' age and sex, tumor location, pathological stage and grade, MMR status, and CMS classification. Multivariate analysis incorporated all variables with a $P$ value below $5 \%$ in univariate analysis. All statistical tests were 2 sided at a $5 \%$ level of significance. Statistical analysis was done using the survival package (version 2.30) in R software (version 2.15.2). This article was written in accordance with reporting recommendations for tumor marker prognostic studies criteria (REMARK) (69). Statistical analysis of quantifications in the mouse study was performed using GraphPad Prism (GraphPad Software). An unpaired, 2-tailed Student's $t$ test was used, except for nuclear size distribution, for which the Kolmogorov-Smirnov test was performed using R software.

Study approval. Mouse experiments were performed in accordance the European Community guidelines (86/609/EEC) and the French National Committee (87/848) for the care and use of laboratory animals and were approved by the regional ethics committee for animal experimentation in Languedoc Roussillon (APAFIS no. 18685-2019012817242507 v3; reference no. 01556.02)

\section{Author contributions}

YG, MG, CP, VP, RT, HT, C. Longobardi, JB, CM, QDC, PF, FBM, BR, C. Lemmers, IZ, KC, SG, RA, EM, and BL conducted experiments and acquired and analyzed data. EM, BL, and $\mathrm{MH}$ designed research studies and analyzed data. YG, FB, JMB, JT, $\mathrm{PS}, \mathrm{EM}, \mathrm{BL}$, and $\mathrm{MH}$ wrote the manuscript. $\mathrm{MH}$ is the principal investigator and managed the mouse experiments. BL was a lead scientist and managed the experiments involving mice. EM was a collaborating scientist and managed experiments involving human samples. For co-first authorship order assignment, YG was involved earlier on in the project, and MG was involved in the revision of the manuscript.

\section{Acknowledgments}

We are grateful for the assistance of the RIO imaging platform, the RHEM and RAM histology and animal experimentation platforms, as well as the IGMM mouse facility in Montpellier. We thank Thierry Gostan for his help with statistical analysis, Ula Hibner for careful reading of the manuscript, and Jan Paul Medema and Susana Prieto for help with establishing organoid cultures. We are grateful to Maguy Del Rio for her help with CCNA2 mRNA expression analysis in patients. The authors acknowledge the Biological Resources Center from the L'Institut du Cancer de Montpellier (ICM) (ICM Biobank number BB 0033 00059). This work was supported by INCA (project 2013-111) and a Site de Recherche Intégrée sur le Cancer (SIRIC) Montpellier Cancer Grant (INCa_Inserm_DGOS_12553 and La Ligue contre le Cancer-Comité 34, to BL). Computing resources for this study were provided by the Datacenter IT and Scientific Computing (DISC) facilities of the Cancer Research Center of Marseille.

Address correspondence to: Michael Hahne or Bénédicte Lemmers, IGMM, UMR5535, 1919 route de Mende, 34293 Montpellier Cedex 5, France. Phone: 33.434.359.639; Email: michael.hahne@ igmm.cnrs.fr (MH); Email: benedicte.lemmers@igmm.cnrs.fr (BL).
1. Dekker E, et al. Colorectal cancer. Lancet. 2019;394(10207):1467-1480.

2. Zlobec I, Lugli A. Prognostic and predictive factors in colorectal cancer. JClin Pathol. 2008;61(5):561-569.

3. Cuyle P-J, Prenen H. Current and future biomarkers in the treatment of colorectal cancer. Acta Clin Belg. 2017;72(2):103-115.

4. Kannarkatt J, et al. Adjuvant chemotherapy for stage II colon cancer: a clinical dilemma. JOncol Pract. 2017;13(4):233-241.

5. McCleary NJ, et al. Personalizing adjuvant therapy for stage II/III colorectal cancer. Am Soc Clin Oncol Educ Book. 2017;37:232-245.

6. Beaugerie L, et al. High risk of anal and rectal cancer in patients with anal and/or perianal Crohn's disease. Clin Gastroenterol Hepatol. 2018;16(6):892-899.

7. $\mathrm{Ma} \mathrm{H}$, et al. Pathology and genetics of hereditary colorectal cancer. Pathology (Phila). 2018;50(1):49-59.

8. Guinney J, et al. The consensus molecular subtypes of colorectal cancer. Nat Med. 2015;21(11):1350-1356.
9. Yasmeen A, et al. E- and A-type cyclins as markers for cancer diagnosis and prognosis. Expert Rev Mol Diagn. 2003;3(5):617-633.

10. Bendris N, et al. Cyclin A2: a genuine cell cycle regulator?. Biomol Concepts. 2012;3(6):535-543.

11. Blanchard JM. To be or not to be a proliferation marker?. Oncogene. 2014;33(8):954-955.

12. Jirawatnotai $S$, et al. A function for cyclin D1 in DNA repair uncovered by protein interactome analyses in human cancers. Nature. 2011;474(7350):230-234.

13. Li Z, et al. Cyclin D1 induction of cellular migration requires $\mathrm{p} 27$ (KIP1). Cancer Res. 2006;66(20):9986-9994.

14. Li Z, et al. Cyclin D1 regulates cellular migration through the inhibition of thrombospondin 1 and ROCK signaling. Mol Cell Biol. 2006;26(11):4240-4256.

15. Li Z, et al. Cyclin D1 functions in cell migration. Cell Cycle. 2006;5(21):2440-2442.

16. McAllister SS, et al. Novel p27(kip1) C-terminal scatter domain mediates Rac-dependent cell migration independent of cell cycle arrest functions. Mol Cell Biol. 2003;23(1):216-228.

17. Arsic N, et al. A novel function for cyclin A2: control of cell invasion via RhoA signalling. JCell Biol. 2012;196(1):147-162.

18. Bendris N, et al. Cyclin A2, a novel regulator of EMT. Cell Mol Life Sci. 2014;71(24):4881-4894.

19. Cheung CT, et al. Cyclin A2 modulates EMT via $\beta$-catenin and phospholipase $C$ pathways. Carcinogenesis. 2015;36(8):914-924.

20. Wang D, et al. Prefoldin 1 promotes EMT and lung cancer progression by suppressing cyclin A expression. Oncogene. 2017;36(7):885-898.

21. Kanakkanthara A, et al. Cyclin A2 is an RNA binding protein that controls Mre11 mRNA translation. Science. 2016;353(6307):1549-1552.

22. Gopinathan L, et al. Loss of Cdk2 and cyclin A2 impairs cell proliferation and tumorigenesis. Cancer Res. 2014;74(14):3870-3879.

23. Kalaszczynska I, et al. Cyclin A is redundant in fibroblasts but essential in hematopoietic and 
embryonic stem cells. Cell. 2009;138(2):352-365.

24. el Marjou F, et al. Tissue-specific and inducible Cre-mediated recombination in the gut epithelium. Genesis. 2004;39(3):186-193.

25. Minoo P, et al. Characterization of rectal, proximal and distal colon cancers based on clinicopathological, molecular and protein profiles. Int JOncol. 2010;37(3):707-718.

26. Taniguchi K, Karin M. IL-6 and related cytokines as the critical lynchpins between inflammation and cancer. Semin Immunol. 2014;26(1):54-74.

27. Parisi L, et al. Macrophage polarization in chronic inflammatory diseases: killers or builders? JImmunol Res. 2018;2018:8917804.

28. Laubitz D, et al. Colonic gene expression profile in NHE3-deficient mice: evidence for spontaneous distal colitis. Am J Physiol Gastrointest Liver Physiol. 2008;295(1):G63-G77.

29. Sobecki M, et al. Cell-cycle regulation accounts for variability in Ki-67 expression levels. Cancer Res. 2017;77(10):2722-2734

30. Todaro M, et al. CD44v6 is a marker of constitutive and reprogrammed cancer stem cells driving colon cancer metastasis. Cell Stem Cell. 2014;14(3):342-356.

31. Afify A, et al. The expression of CD44v6 in colon: from normal to malignant. Ann Diagn Pathol. 2016;20:19-23.

32. Hayashi MT, Karlseder J. DNA damage associated with mitosis and cytokinesis failure. Oncogene. 2013;32(39):4593-4601.

33. van den Bosch M, et al. The MRN complex: coordinating and mediating the response to broken chromosomes. EMBO Rep. 2003;4(9):844-849.

34. Rein K, Stracker TH. The MRE11 complex: an important source of stress relief. Exp Cell Res. 2014;329(1):162-169.

35. Karin M, Clevers H. Reparative inflammation takes charge of tissue regeneration. Nature. 2016;529(7586):307-315.

36. Yui S, et al. YAP/TAZ-dependent reprogramming of colonic epithelium links ECM remodeling to tissue regeneration. Cell Stem Cell. 2018;22(1):35-49.

37. Merenda A, et al. Wnt signaling in 3D: recent advances in the applications of intestinal organoids. Trends Cell Biol. 2020;30(1):60-73.

38. De Robertis M, et al. The AOM/DSS murine model for the study of colon carcinogenesis: From pathways to diagnosis and therapy studies. JCarcinog. 2011;10:9.
39. Erben U, et al. A guide to histomorphological evaluation of intestinal inflammation in mouse models. Int JClin Exp Pathol. 2014;7(8):4557-4576.

40. Hydbring P, et al. Non-canonical functions of cell cycle cyclins and cyclin-dependent kinases. Nat Rev Mol Cell Biol. 2016;17(5):280-292.

41. Chen W, et al. Proteasome-mediated destruction of the cyclin a/cyclin-dependent kinase 2 complex suppresses tumor cell growth in vitro and in vivo. Cancer Res. 2004;64(11):3949-3957.

42. Clevers $\mathrm{H}$, et al. Stem cell signaling. An integral program for tissue renewal and regeneration: Wnt signaling and stem cell control. Science. 2014;346(6205):1248012.

43. Schwitalla $\mathrm{S}$, et al. Intestinal tumorigenesis initiated by dedifferentiation and acquisition of stem-celllike properties. Cell. 2013;152(1-2):25-38.

44. Gupta PB, et al. Cancer stem cells: mirage or reality? Nat Med. 2009;15(9):1010-1012.

45. Hanahan D, Weinberg RA. Hallmarks of cancer: the next generation. Cell. 2011;144(5):646-674.

46. Kim SI, et al. Cyclin-dependent kinase 2 regulates the interaction of Axin with beta-catenin. Biochem Biophys Res Commun. 2004;317(2):478-483.

47. Petersen BO, et al. Phosphorylation of mammalian CDC6 by cyclin A/CDK2 regulates its subcellular localization. EMBO J.1999;18(2):396-410.

48. Kabeche L, Compton DA. Cyclin A regulates kinetochore microtubules to promote faithful chromosome segregation. Nature. 2013;502(7469):110-113.

49. Ganem NJ, Pellman D. Linking abnormal mitosis to the acquisition of DNA damage. J. Cell Biol. 2012;199(6):871-881.

50. Lee Y-C, et al. High expression of phospho-H2AX predicts a poor prognosis in colorectal cancer. Anticancer Res. 2015;35(4):2447-2453.

51. Li J-A, et al. Cyclin A2 regulates symmetrical mitotic spindle formation and centrosome amplification in human colon cancer cells. Am J Transl Res. 2018;10(8):2669-2676.

52. Georgakilas AG, Kotsinas A. Editorial: DNA damage and inflammation under stress. Front Genet. 2017;8:152.

53. Fordyce CA, et al. Cell-extrinsic consequences of epithelial stress: activation of protumorigenic tissue phenotypes. Breast Cancer Res. 2012;14(6):R155.

54. Fordyce $\mathrm{C}$, et al. DNA damage drives an activin a-dependent induction of cyclooxygenase- 2 in premalignant cells and lesions. Cancer Prev Res (Phila).
2010;3(2):190-201.

55. Jayasingam SD, et al. Evaluating the polarization of tumor-associated macrophages into M1 and M2 phenotypes in human cancer tissue: technicalities and challenges in routine clinical practice. Front Oncol. 2020;24;9:1512.

56. Marafini I, et al. TGF-Beta signaling manipulation as potential therapy for IBD. Curr Drug Targets. 2013;14(12):1400-1404.

57. Zanconato F, et al. YAP/TAZ at the roots of cancer. Cancer Cell.2016;29(6):783-803.

58. Yuen $\mathrm{H}-\mathrm{F}$, et al. TAZ expression as a prognostic indicator in colorectal cancer. PLoS One. 2013;8(1):54211.

59. Handa K, et al. Expression of cell cycle markers in colorectal carcinoma: superiority of cyclin A as an indicator of poor prognosis. Int JCancer. 1999;84(3):225-233.

60. Bahnassy AA, et al. Cyclin A and cyclin D1 as significant prognostic markers in colorectal cancer patients. BMC Gastroenterol. 2004;4:22.

61. Nozoe T, et al. Clinicopathologic significance of cyclin A expression in colorectal carcinoma. J Exp Clin Cancer Res. 2004;23(1):127-133.

62. Li J-Q, et al. Cyclin A correlates with carcinogenesis and metastasis, and p27(kip1) correlates with lymphatic invasion, in colorectal neoplasms. Hum Pathol. 2002;33(10):1006-1015.

63. Bray NL, et al. Near-optimal probabilistic RNA-seq quantification. Nat Biotechnol. 2016;34(5):525-527.

64. Dobin A, et al. STAR: ultrafast universal RNA-seq aligner. Bioinformatics. 2013;29(1):15-21.

65. Irizarry RA, et al. Exploration, normalization, and summaries of high density oligonucleotide array probe level data. Biostatistics. 2003;4(2):249-264.

66. Bertucci F, et al. EndoPredict predicts for the response to neoadjuvant chemotherapy in ER-positive, HER2-negative breast cancer. Cancer Lett. 2014;355(1):70-75.

67. Eide PW, et al. CMScaller: an R package for consensus molecular subtyping of colorectal cancer pre-clinical models. Sci Rep. 2017;7(1):16618.

68. Hochberg Y, Benjamini Y. More powerful procedures for multiple significance testing. Stat Med. 1990;9(7):811-818.

69. McShane LM, et al. REporting recommendations for tumor MARKer prognostic studies. Nat Clin Pract Urol. 2005;2(8):416-422. 